\title{
What the Brain Stem Tells the Frontal Cortex. II. Role of the SC-MD-FEF Pathway in Corollary Discharge
}

\author{
Marc A. Sommer and Robert H. Wurtz \\ Laboratory of Sensorimotor Research, National Eye Institute, National Institutes of Health, Bethesda, Maryland 20892-4435
}

Submitted 31 July 2003; accepted in final form 15 October 2003

Sommer, Marc A. and Robert H. Wurtz. What the brain stem tells the frontal cortex. II. Role of the SC-MD-FEF pathway in corollary discharge. J Neurophysiol 91: 1403-1423, 2004. First published October 22,$2003 ; 10.1152 / j n .00740 .2003$. One way we keep track of our movements is by monitoring corollary discharges or internal copies of movement commands. This study tested a hypothesis that the pathway from superior colliculus (SC) to mediodorsal thalamus (MD) to frontal eye field (FEF) carries a corollary discharge about saccades made into the contralateral visual field. We inactivated the MD relay node with muscimol in monkeys and measured corollary discharge deficits using a double-step task: two sequential saccades were made to the locations of briefly flashed targets. To make second saccades correctly, monkeys had to internally monitor their first saccades; therefore deficits in the corollary discharge representation of first saccades should disrupt second saccades. We found, first, that monkeys seemed to misjudge the amplitudes of their first saccades; this was revealed by systematic shifts in second saccade end points. Thus corollary discharge accuracy was impaired. Second, monkeys were less able to detect trial-by-trial variations in their first saccades; this was revealed by reduced compensatory changes in second saccade angles. Thus corollary discharge precision also was impaired. Both deficits occurred only when first saccades went into the contralateral visual field. Single-saccade generation was unaffected. Additional deficits occurred in reaction time and overall performance, but these were bilateral. We conclude that the SC-MD-FEF pathway conveys a corollary discharge used for coordinating sequential saccades and possibly for stabilizing vision across saccades. This pathway is the first elucidated in what may be a multilevel chain of corollary discharge circuits extending from the extraocular motoneurons up into cerebral cortex.

\section{N T R O D U C T I O N}

Generating movements is a critical part of everyday life, and it is perhaps equally important that we keep track of movements as we make them. Monitoring our actions helps us to execute complex behaviors rapidly and to ignore spurious sensory inputs caused by movements. Information about movements comes from sensory receptors, including those in muscles, and from internal records of movement known as corollary discharge.

The principle of corollary discharge (Sperry 1950) is that when a brain region sends a motor command downstream, it simultaneously sends a copy upstream to be used as information about the movement (Fig. 1A). As a way to monitor movements, corollary discharge (or efference copy) (von Holst and Mittelstaedt 1950) has two main advantages over sensory cues: it provides information even before movement onset and

Address for reprint requests and other correspondence: M. A. Sommer, Bldg 49, Room 2A50, MSC 4435, NEI, NIH, 9000 Rockville Pike, Bethesda, MD 20892-4435 (E-mail: mas@1sr.nei.nih.gov). it does not rely on the integrity of sensory receptors. The concept of corollary discharge has helped to answer questions as diverse as how crickets chirp without damaging their hearing (Poulet and Hedwig 2002) and why we can tickle others but not ourselves (Blakemore et al. 2000). Impaired corollary discharge may contribute to pathologies including schizophrenia (Feinberg and Guazzelli 1999; Ford et al. 2001) and may underlie many of the sensory and motor deficits that follow brain damage (Angel 1980; Baizer et al. 1999; Duhamel et al. 1992b; Gaymard et al. 1994; Haarmeier et al. 1997; Heide et al. 1995; Rafal 1994; Versino et al. 2000). Neurons in several parts of the primate brain seem to receive corollary discharge because their visual responses change just prior to movements (Duhamel et al. 1992a; Reppas et al. 2002; Richmond and Wurtz 1980; Thiele et al. 2002; Tolias et al. 2001; Umeno and Goldberg 1997).

In the accompanying paper (Sommer and Wurtz 2004b), we described a pathway from superior colliculus (SC) to frontal eye field (FEF; Fig. 1B) that transmits a variety of signals including bursts of activity just prior to contraversive saccades (i.e., saccades made into the contralateral visual field). This activity provides information about when a saccade will occur and where it will go. Because it travels away from the eye muscles and up to the FEF, a cortical region involved in visual analysis and saccadic planning (Schall 1997), we hypothesized that the activity was a corollary discharge.

The goal of the present study was to test this hypothesis by examining the consequences of inactivating the pathway. Signals sent from the SC to the FEF are relayed by neurons in the mediodorsal thalamus (MD), something, from an experimental point of view, that is highly fortuitous; shutting off those neurons should interrupt signal flow in the pathway without directly affecting either the pathway's source, the SC, or its target, the FEF (Fig. 1B). We injected the $\mathrm{GABA}_{\mathrm{A}}$ agonist muscimol at the sites of previously recorded MD relay neurons (Fig. 1C) (Lomber 1999). This should temporarily inactivate the neurons because $\mathrm{GABA}_{\mathrm{A}}$ receptors are found throughout MD (Steriade et al. 1997).

We reasoned that if the pathway conveys corollary discharge signals, then during MD inactivation, a monkey should be impaired at keeping track of its contraversive saccades. One task that requires the internal monitoring of saccades is the double-step task (Becker and Jürgens 1979; Hallett and Lightstone 1976; Mays and Sparks 1980), which has been used extensively in prior studies to test for corollary discharge deficits (Duhamel et al. 1992b; Gaymard et al. 1994; Heide et

\footnotetext{
The costs of publication of this article were defrayed in part by the payment of page charges. The article must therefore be hereby marked "advertisement" in accordance with 18 U.S.C. Section 1734 solely to indicate this fact.
} 
A

$$
\text { Corollary Discharge }
$$

(information about saccades)

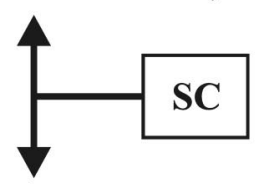

Motor Command

(generation of saccades)

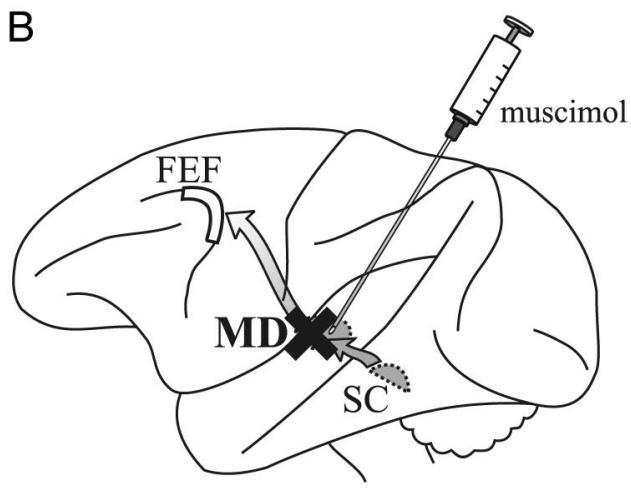

C

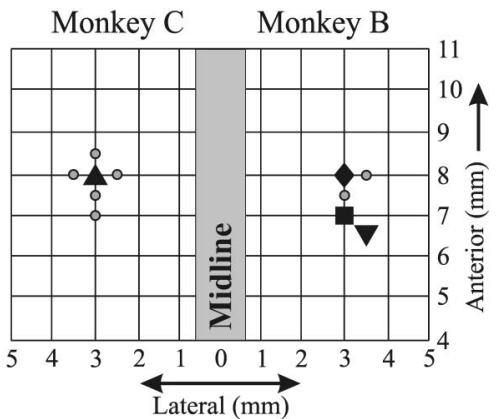

FIG. 1. Hypothesis and methods. A: we hypothesized that the superior colliculus (SC) sends a corollary discharge upstream at the same time it sends a motor command downstream. $B$ : to test our hypothesis we interrupted the signals (X) flowing upstream through the pathway from SC to mediodorsal thalamus (MD) to the frontal eye field (FEF) and studied the monkey's behavior. The interruption was achieved by injecting muscimol at the sites of previously recorded MD relay neurons. $C$ : injection sites relative to recording sites. Previously (Sommer and Wurtz 2004b) we found MD relay neurons at sites marked with a dot or polygon. Here we performed injections at the sites marked with polygons as follows: $\boldsymbol{\Delta}$, injections $1-3$ (all muscimol); $\bullet$, injection 4 (muscimol) and 7 (saline); $\boldsymbol{\square}$, injection 5 (muscimol); $\mathbf{v}$, injection 6 (saline). Table 1 provides details of each injection. Note that the injection sites in the 2 monkeys were in opposite hemispheres, but for consistency throughout this paper we will depict all contraversive saccades (relative to the injection site) as rightward.

al. 1995). In this task, subjects make saccades sequentially to the locations of two flashed targets (Fig. 2A). To make the second saccade correctly, a subject must keep track of where its first saccade goes; thus deficits in monitoring first saccades should disrupt second saccades. Visual feedback is unavailable because the targets disappear before the eyes move, and information from eye muscle proprioception seems to be negligible (Bridgeman 1995; Guthrie et al. 1983; Lewis et al. 2001; Steinbach 1987). Keeping track of the first saccade should depend primarily on monitoring the corollary discharge of that saccade.

In principle, MD inactivation could impair the accuracy or the precision of corollary discharge. The concepts of accuracy and precision are classically illustrated using the example of target shooting (Fig. 2B, top). Relative to a shooter's normal behavior (left), he or she would be suffering an accuracy deficit if the shot cluster shifted systematically (middle) or a precision deficit if the shots became more scattered (right). Analogously, corollary discharge is an attempt to "hit the bull's-eye"-the brain wants to create a corollary discharge signal that matches a saccade. Consider three trials of making a saccade (Fig. 2B, bottom). The saccade is constant from trial to trial, but the corollary discharge representation of it may vary. Relative to how well the corollary discharge signals normally represent the saccade (left), they would suffer an accuracy deficit if they started to systematically misrepresent the saccade, e.g., if they became shorter on average (middle), or a precision deficit if they started to vary more (right).

These corollary discharge deficits, although not directly observable, should be detectable in the double-step task through their influence on second saccades. To illustrate this, Fig. $2 C$ depicts a monkey's internal estimate of its behavior (top) and its actual behavior (bottom). The monkey's goal is to make a second saccade that reaches the second target location, and to do this, it must monitor its corollary discharge representation of the first saccade. From trial to trial, the monkey will monitor its corollary discharge signals and make adjustments so as to keep its second saccade end-points constant (Fig. 2C, top), but because the first saccade does not really change, these adjustments will actually introduce errors into the second saccade end points (Fig. 2C, bottom). Compared with the normal behavior (Fig. $2 C$, bottom left), if there is a corollary discharge accuracy deficit, the second saccade end points will shift systematically (bottom middle), and if there is a corollary discharge precision deficit, these end points will become more scattered (bottom right).

Finally, a critical expectation is that these changes in second saccades should be lateralized, occurring only in trials involving contraversive first saccades. This is because the putative corollary discharge signal (presaccadic activity in the SC-MDFEF pathway) is related almost exclusively to contraversive saccades (Sommer and Wurtz 2004b).

We found that our hypothesis was supported: MD inactivation caused contralateralized deficits in both the accuracy and the precision of corollary discharge. This provides direct evidence that the presaccadic signals sent from SC to FEF represent corollary discharge information about saccades. A brief report regarding the accuracy deficit was published previously (Sommer and Wurtz 2002).

\section{METHODS}

\section{Monkeys}

We used monkeys $B$ and $C$ from our study of MD relay neurons (Sommer and Wurtz 2004b). Both monkeys had been implanted with scleral search coils for measuring eye position, a post for holding the head, and chambers for accessing the brain. All procedures were approved by the Institute Animal Care and Use Committee and complied with Public Health Service Policy on the humane care and use of laboratory animals. 

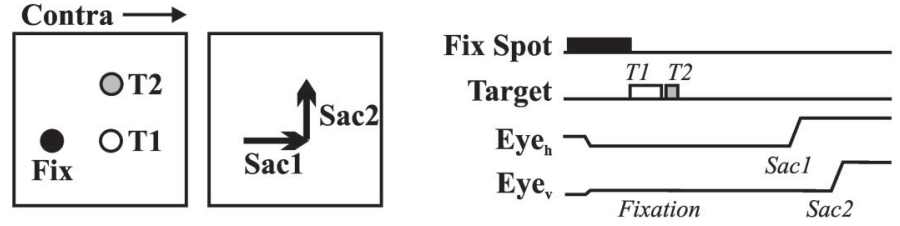

B

Normal
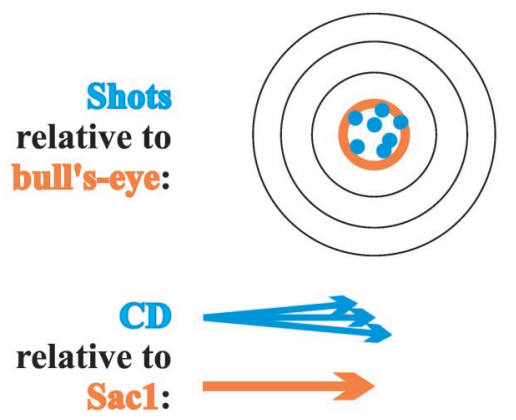

C

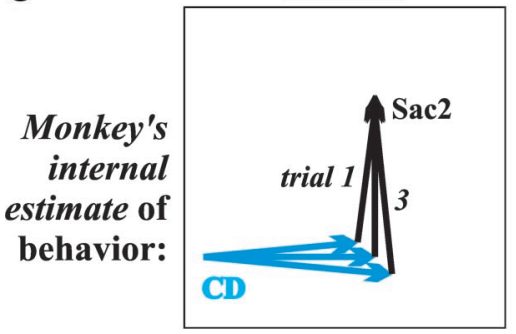

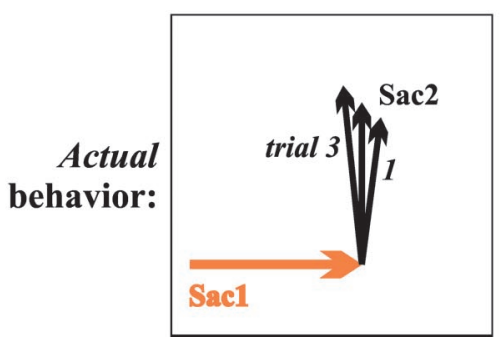

Accuracy Deficit
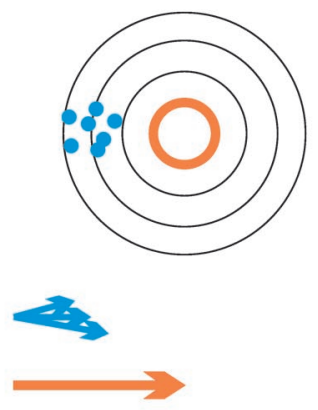

Accuracy Deficit
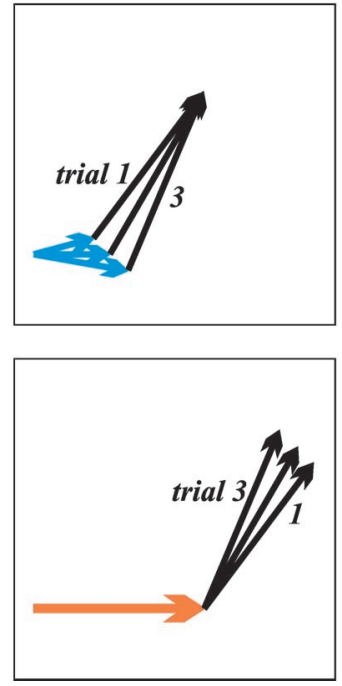

Precision Deficit
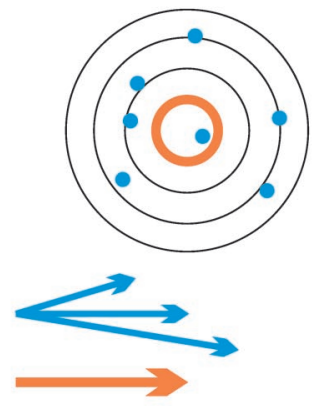

Precision Deficit
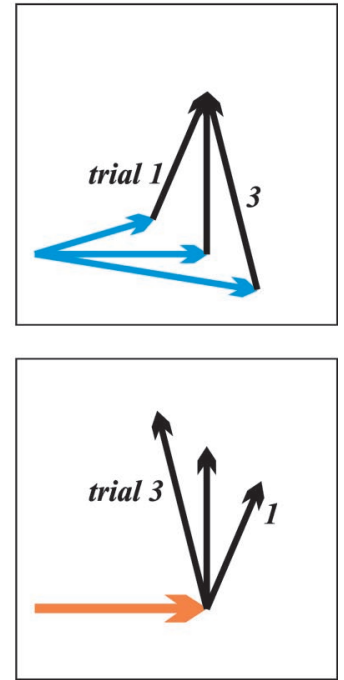

FIG. 2. Detecting corollary discharge deficits using the double-step task. A: spatial (left) and temporal (right) aspects of the task. Monkeys initially foveated a fixation spot (Fix) and then 2 targets (T1 and T2) appeared sequentially in the periphery. The task was to make 2 saccades (Sac1 and Sac2) to the locations of the extinguished targets. Contra, contraversive; Eye $_{\mathrm{h}}$ and $\mathrm{Eye}_{\mathrm{v}}$, horizontal and vertical components of the eye position. $B$ : accuracy and precision deficits. Normal behavior (left) is compared with deficits in accuracy (middle) or precision (right). Top: normal and impaired sharp-shooting. In multiple trials, shots (blue dots) are made in attempt to hit the bull's-eye (orange ring). Bottom: normal and impaired internal monitoring of saccades. In multiple trials, the brain creates corollary discharge signals (CD, blue arrows) in attempt to represent a saccade (orange arrow). $C$ : normal and impaired double-step behavior. The monkey's internal estimate of its behavior (top) is contrasted with its actual behavior (bottom). Relative to the normal behavior (left), 2nd saccades should change considerably during a corollary discharge accuracy deficit (middle) or precision deficit (right). Second saccades from 2 of the 3 hypothetical trials are labeled (trials 1 and 3) to help illustrate how the monkey's reliance on its CD signals (top) causes the various patterns of second saccades (bottom). See INTRODUCTION for details.

\section{Injection sites}

We injected at a site only if it met three criteria: past recordings at the site must have yielded identified MD relay neurons (i.e., only the sites shown in Fig. $1 C$ were considered), recordings the day before injecting at the site had to demonstrate strong multiunit activity, indicating that the site was still healthy, and electrical stimulation at the site had to evoke saccades because we used changes in stimulation-evoked saccades to assess whether muscimol was successfully injected (see following text). At each site, we injected either muscimol dissolved in saline $(5 \mu \mathrm{g} / \mu \mathrm{l})$ or saline alone as a control for incidental effects (e.g., pressure or dilution) that might be caused by liquid infusion. Table 1 lists all the experiments (1st column) and the agent injected in each (2nd column). One site in monkey C (Fig. 1C, left, $\mathbf{\Delta}$ ) was extraordinarily well suited for inactivation, as it met all three criteria and, moreover, was where we had found a particularly rich concentration of MD relay neurons [cf. Fig. 2A, left, hatched circle, in Sommer and Wurtz (2004b)]; the surrounding sites were less attractive for injecting (less robust neuronal activity, no saccades evoked, or both). We restricted our injections in monkey $C$ to this one site to minimize inactivation of MD areas that did not contain relay neurons. As shown in Table 1, we performed different tests during each inactivation at this site. In monkey $B$, we injected at three sites that met our criteria $(\bullet, \mathbf{\square}$, and $\nabla$ in Fig. $1 C$, right). We terminated the study once we ran out of sites that met our criteria for inactivation. Although the number of inactivation sites was limited, it was nonetheless sufficient to demonstrate significant, lateralized corollary discharge deficits in both the pooled data and in each monkey individually as reported in the RESULTS.

\section{Injection procedure}

We inserted a 30-gauge cannula through a 23-gauge guide tube into the brain until the cannula tip was at the depth of previously recorded $\mathrm{MD}$ relay neurons. The other end of the cannula was attached to a $10-\mu 1$ Hamilton syringe, and we injected muscimol manually in boluses of $0.2 \mu \mathrm{l}$ every $30 \mathrm{~s}$. A major technical issue was ensuring that muscimol was ejected from the cannula. We aborted the experiment if resistance was felt that might indicate a plugged cannula tip or if muscimol ejection was not verified by changes in stimulation-evoked saccades. Four injections ( 2 in each monkey) were aborted for these reasons and are not included in this report. An insulated wire threaded through the cannula, with its exposed tip extending $500 \mu$ beyond the 
TABLE 1. Summary of experiments

\begin{tabular}{|c|c|c|c|c|}
\hline $\begin{array}{l}\text { Injection Number } \\
\text { (Monkey) }\end{array}$ & $\begin{array}{c}\text { Agent } \\
\text { (Amount) }\end{array}$ & $\begin{array}{l}\text { Experiments } \\
\text { Performed }\end{array}$ & $\begin{array}{l}\text { Ambient } \\
\text { Illumination }\end{array}$ & $\begin{array}{c}\text { Target } \\
\text { Configuration* }\end{array}$ \\
\hline $1(C)$ & Muscimol (2.4) & Double-step & Light & A \\
\hline $2(C)$ & & Double-step & Dark & A \\
\hline \multirow[t]{3}{*}{$3(C)$} & Muscimol (1.6) & Double-step & Light & B \\
\hline & & Double-step & Dark & B \\
\hline & & Single-step & Light & A \\
\hline \multirow[t]{2}{*}{$5(B)$} & Muscimol (1.6) & Double-step & Dark & $\mathrm{C}$ \\
\hline & & Single-step & Dark & B \\
\hline $6(B)$ & Saline (4.4) & Double-step & Dark & $\mathrm{C}$ \\
\hline $7(B)$ & Saline (4.4) & Double-step & Dark & $\mathrm{C}$ \\
\hline
\end{tabular}

Agent amounts are in microliters. * Target configurations represented by the letters A-C are shown in Fig. 4.

cannula tip, allowed us to electrically stimulate at each injection site (Crist et al. 1988). We found that stimulating at relay neuron sites (biphasic cathodal-anodal pulses, $0.25 \mathrm{~ms} / \mathrm{ph}$ ase, 350-Hz, $150-\mathrm{ms}$ trains) often evoked saccades having scattered vectors (not stereotyped as when stimulating SC or FEF) after long latencies (mean: 96 ms after stimulation onset) and at moderate current thresholds (mean: $36 \mu \mathrm{A}$ ); other stimulation results are beyond the scope of this report. Our indicator of successful muscimol ejection from the cannula was a marked increase in current threshold (typically $>2$ times the initial level) around 20-30 min after the injection, when muscimol typically begins having strong effects (e.g., Aizawa and Wurtz 1998). This rise in threshold may have been due in part to tissue displacement; we did not systematically compare the effects of muscimol versus saline injection to test this. Regardless of the exact mechanism(s) causing it, the increased threshold confirmed that liquid had been ejected into the brain. The increase in current threshold marked the start of the "during" inactivation period discussed throughout this report. The current threshold increase always persisted throughout the session, paralleling the multiple hour time course of muscimol (e.g., see Lomber 1999).

\section{General protocol}

The day before an injection we recorded at the targeted site to ensure that neurons were active there and collected "before" behavioral data. The day of the injection we collected baseline stimulation data, injected muscimol and collected stimulation data to verify its delivery, and collected during behavioral data. On a later day (usually the next day) we collected "after" behavioral data to measure recovery.

\section{Experimental apparatus}

During each experiment a monkey faced a tangent screen onto which visual stimuli were projected. Ambient illumination was dim light or darkness. In dim-light experiments, we used an LCD projector that even at its darkest setting illuminated the tangent screen with a diffuse gray background $\left(0.1 \mathrm{~cd} / \mathrm{m}^{2}\right)$. Visual stimuli were a blue fixation spot and red targets $\left(0.6 \mathrm{~cd} / \mathrm{m}^{2}\right)$. In darkness experiments, we used lasers to produce red dots for the fixation spot and targets. There was no other source of light in the room during each trial, and between trials a lamp was illuminated to prevent dark-adaptation. We sampled eye position and recorded task events every $1 \mathrm{~ms}$.

\section{Double-step task}

The purpose of this task was to test whether MD inactivation impaired corollary discharge (see INTRODUCTION). A monkey fixated a spot, the spot disappeared, and two targets briefly appeared in se- quence (Fig. 2A). To receive reward (a drop of water), the monkey had to make two sequential saccades to the locations of the targets. The timings of target presentation (specifically, the 1st target duration, the gap between offset of the 1st target and onset of the 2nd, and the 2nd target duration) varied depending on the target pair and the monkey because we always fine-tuned the timings until errors were minimized. For example, if for a certain target pair a monkey often erred by making its first saccade to target 2 instead of target 1 , we lengthened the target 1 duration, shortened the target 2 duration, and changed the gap between targets 1 and 2 until the errors were minimized. The only constraint was that the overall sum of the target 1 duration, the gap between targets 1 and 2, and the target 2 duration be less than $\sim 180$ $\mathrm{ms}$, approximately the minimum reaction time of first saccades relative to target 1 onset. This constraint was imposed because we required all visual stimuli to disappear before the first saccade began so that there would be no visual feedback from the targets that might inform the monkey whether the first saccade was made or where it landed. In practice, the typical target 1 durations were $\sim 130 \mathrm{~ms}$, the gap was $\sim 20 \mathrm{~ms}$, and the target 2 duration was $\sim 30 \mathrm{~ms}$. On-line, we used a real-time saccadic velocity detector to abort trials in which the first saccade began too early; off-line, we inspected every trial to make sure that the more precise saccade detection algorithm in our analysis program agreed with this on-line analysis, and we eliminated further trials as needed. Spatially, we randomized a variety of target pairs as will be explained in RESULTS.

\section{Single-step task}

The purpose of this task was to see if inactivation affected the generation of saccades. A monkey fixated a spot, the spot disappeared, a gap of $150 \mathrm{~ms}$ ensued, and then a single target appeared. The monkey had to make one saccade directly to the target. In a "targetpresent" version of this task, once the peripheral target appeared, it stayed lit until after the monkey made a saccade to it. In contrast, a "target-absent" version involved carefully chosen parameters so that the target presentation closely matched the presentation of target 2 in the double-step task. We did this to see how monkeys responded to a flashed target when there was no intervening first saccade, i.e., when corollary discharge was not a factor. The 150-ms gap approximated the target 1 duration plus the gap between targets 1 and 2 in the double-step task, and when the target appeared, it stayed lit for the same length of time (typically $\sim 30 \mathrm{~ms}$ ) that target 2 would have appeared in the double-step task. Because of the brief target flash, the saccade did not begin until $\sim 120 \mathrm{~ms}$ after the target disappeared (saccadic reaction times were $\sim 150 \mathrm{~ms}$ ), and thus the saccade went to the remembered location of the target. This memory requirement was nearly identical to that required for making saccades to target 2 in the double-step task. Spatially, we randomized a variety of target locations (see RESULTS). 


\section{Analysis}

On-line, in the double-step task we used rather large virtual windows around the targets for judging whether saccades were correct, because we did not want monkeys to receive feedback (due to loss of reward) that their behavior had changed if their second saccades became drastically different due to corollary discharge impairment. Typically the windows were $10^{\circ}$ horizontally by $10^{\circ}$ vertically around target 1 and $20 \times 20^{\circ}$ around target 2 . Initial fixation windows were typically $5 \times 5^{\circ}$ and sometimes a bit larger; this was somewhat liberal but necessary because our monkeys seemed to find it difficult to sustain their fixation on the laser spots in total darkness, particularly when fixation was required at eccentric locations. In the single-step task, we used smaller windows, typically $5 \times 5^{\circ}$, around the target.

Off-line, we detected saccades in the data with software that used a template-matching algorithm. We manually reviewed every trial to verify the saccade detection and to remove error trials. Correct trials were those in which the first and second saccades landed in welldefined clusters of end points within a few degrees (exact distance depended on the target configuration) of the first or second target location, respectively. Most error trials (see Fig. 13C) were obvious by inspection and included $\sim 20-35 \%$ of trials in which the first saccade went to the second target, $\sim 5-10 \%$ of trials in which the first saccade was correct but the second saccade did not occur or went to the wrong location, $\sim 10 \%$ of trials in which the first saccade went nowhere near the first or second target, and very rare saccades that were curved or otherwise atypical (e.g., the strongly curved upward 2nd saccade in Fig. 3A, bottom). The only ambiguity in identifying error trials came from first saccades that landed between the first and second targets ("averaging" saccades); we decided to accept such saccades as correct if they landed closer to the first than the second target.

All statistical tests used a criterion of $P<0.05$, Bonferroni cor- rected as needed if there were multiple comparisons on the same data set. Unless explicitly noted, throughout this study we compared data sets using Student's $t$-test, if judged normal by the KolmogorovSmirnov test and of equal variance by the Levene Median test, or else the Mann-Whitney rank sum test, and we analyzed correlations using Pearson's test.

\section{RES ULTS}

\section{Example injections}

Figure 3 shows data from an example inactivation. The monkey had to make a rightward saccade and then an upward saccade in response to two flashed targets. Figure $3 A$, top, shows all trials performed by the animal before MD was inactivated. Most saccades were made in the appropriate sequence, going toward the first target and then the second. During MD inactivation (Fig. 3A, bottom), the monkey's behavior was nearly the same except that the second, upward saccades seemed to land, on average, at more contralateral locations. We selected from these raw data only trials in which the monkey made correct sequences (Fig. 3B; see METHODS), and in Fig. $3 C$, we summarize these correct trials by showing the means and SDs of the initial fixations, first saccade end points, and second saccade end points before and during inactivation. The most obvious effect of the inactivation seemed to be a roughly horizontal shift in second saccade end points. We examined this quantitatively by testing whether the end points were significantly different during versus before inactivation along the horizontal dimension and, for comparison, along the

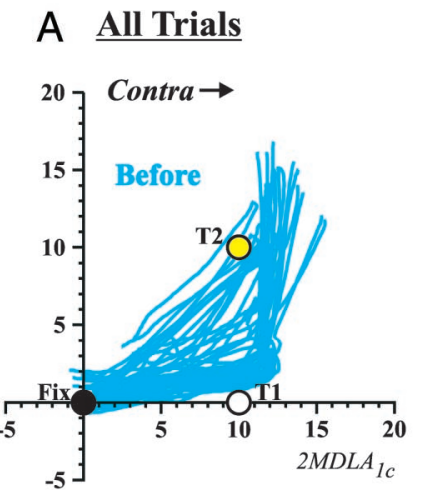

\section{B Correct Trials Only}
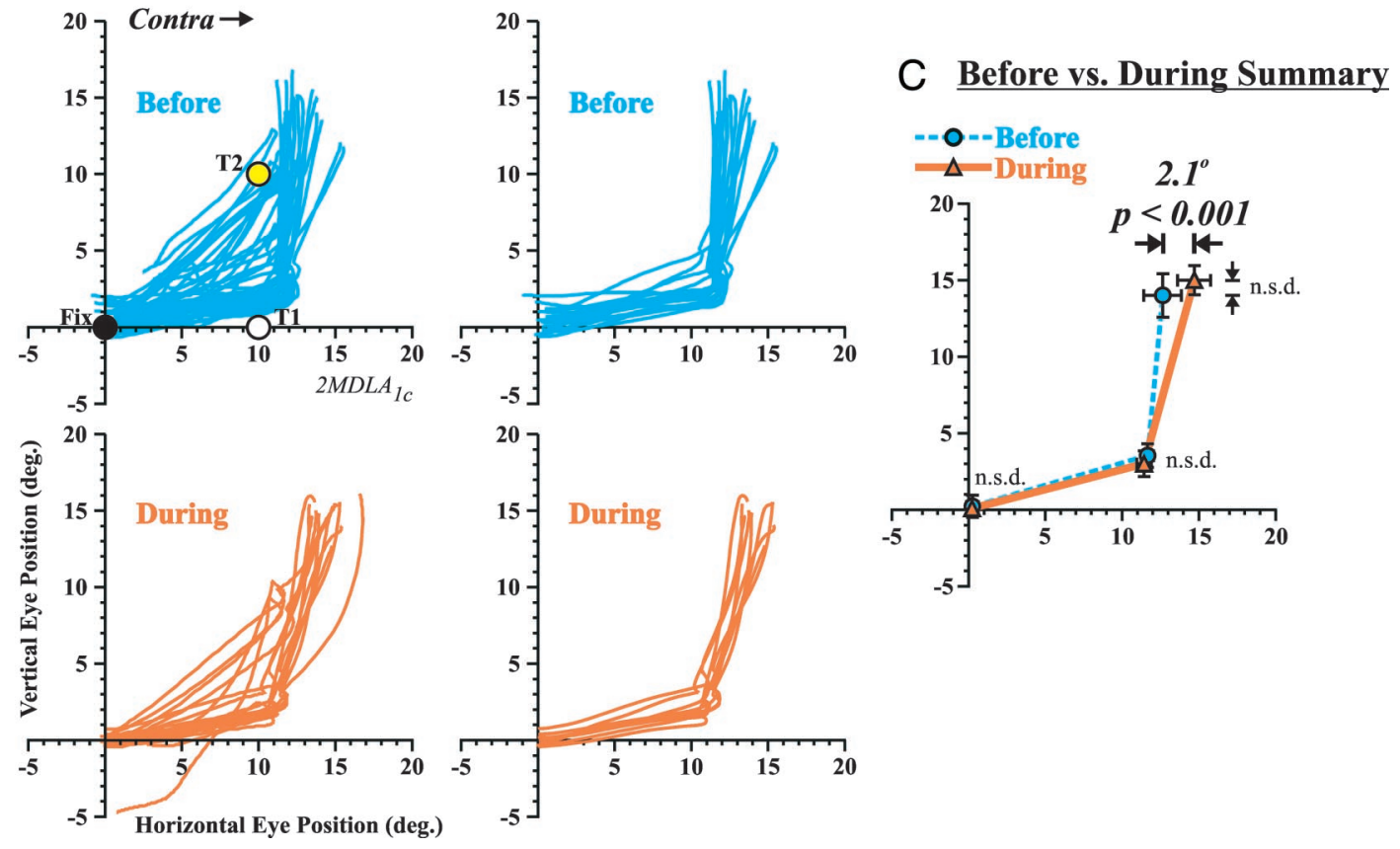

FIG. 3. Example data from the double-step task before (blue) and during (orange) MD inactivation. Superimposed are saccades from all trials $(A)$ and correct trials only $(B)$. The dots represent the fixation spot (Fix) and target locations (T1 and T2). $C$ : summary of the correct trials before and during inactivation, showing the means \pm SDs of initial fixation locations, 1st saccade end points, and 2 nd saccade end points. The only significant change during inactivation was a contraversive shift in 2 nd saccade end points. n.s.d., not significantly different. The label " $2 \mathrm{MDLA}_{1 \mathrm{c}}$ " in $A$ identifies the data according to the following code: the 1 st 5 characters correspond to the five columns of Table 1 (injection 2, Muscimol, Double-step task, Light, configuration $A$ ) and the subscript identifies the specific target pair used (target pair 1, contralateral). Diagrams of target configurations and specific target pairs are shown in Fig. 4A. This labeling system is used throughout the paper. 
vertical dimension (criterion level $P<0.025$, Bonferroni corrected from $P<0.05$ due to testing in 2 directions). Figure $3 C$ shows that in this example case, there was indeed a significant contraversive shift in second saccade end points. In contrast, neither the initial fixations nor the first saccade end points shifted in either the horizontal or vertical direction.

We note that in Fig. 3 and in subsequent examples it is obvious that the saccadic sequences often were somewhat inaccurate relative to the actual target locations. This was because, first, upward shifts sometimes occurred as a normal behavior of the monkeys. Such shifts are common when saccades are made to remembered target locations in darkness (Gnadt et al. 1991), and in our experience, this happens even in dim light for some monkeys (e.g., monkey $C$ ). Second, we purposely avoided over-training the monkeys. If they were to establish and execute programmed sequences of saccades as a rote response to a particular target pair stimulus (e.g., Kowler 1990), they could then perform the task without continuously monitoring their first saccades. We therefore changed target configurations frequently and allowed monkeys to practice on them for only a few days, at most, before each injection experiment.

Figure $4 A$ shows the three target configurations we used (left) and example saccadic sequences generated by the monkeys in response to each (right). Each configuration involved horizontal first saccades followed by vertical or oblique second saccades. In each injection experiment, we used a single configuration with all the target pairs of that configuration randomized by trial. The first two configurations, $A$ and $B$ (Fig. $4 A$, top and middle), involved a central fixation point and centrifugal first saccades. The advantage of these two configurations was that the sequence was unpredictable at the start of fixation; the disadvantage, however, was that second saccades began at an eccentric location. Second saccades were the most crucial movements for us to measure, so it would be preferable if they started at the center so that they could be measured optimally (maximum linearity of the eye coil system was in the center) and so that their trajectories would be unaffected by mechanical limits at the orbital extremes. Thus in a third configuration, $C$ (Fig. 4A, bottom), we used centripetal first saccades and second ones starting at the center of the screen. The disadvantage of this configuration was that it introduced predictability into the paradigm (the initial fixation location provided information as to whether the sequence would be rightward or leftward), so to counter this problem, we introduced extra randomness by adding many more second target locations. Each configuration, $A, B$, or $C$, resulted in a different pattern of saccadic sequences that approximately matched the arrangement of targets in the configuration (Fig. 4A, right), and thus it appeared that the monkeys did try to perform the sequences correctly even though hampered by vertical upshifts and only limited training, as noted in the preceding text. Second saccades clearly were attempts to reach the second target locations and were not just default saccades.

Figure 5 shows examples of inactivation results obtained using the various target configurations. The data in Fig. 5A were collected using configuration $A$ and demonstrate that second saccade end-point shifts occurred for downward sequences as well as upward ones (for clarity, however, we restrict all other examples in this report to upward sequences). Figure $5 B$ shows an example obtained using configuration $B$,

\section{A}

Double-Step Task Configurations
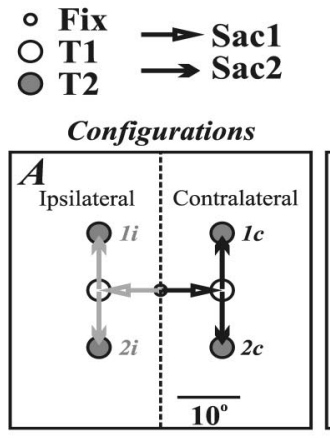

Example Sequences
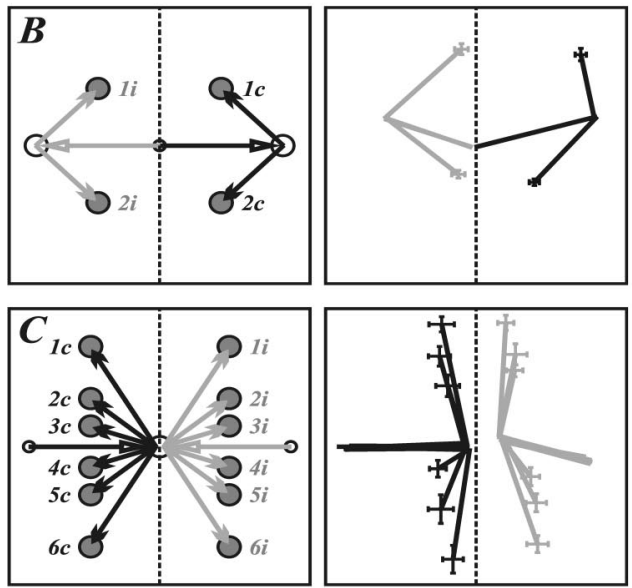

B

Single-Step Task Configurations

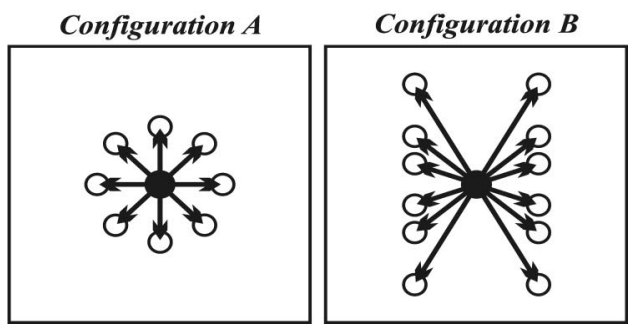

FIG. 4. The target configurations used in this study. A, left: double-step configurations $A$ (top), $B$ (middle), and $C$ (bottom). Each configuration comprised several target pairs that shared a common 1st target in the contra- or ipsilateral hemifield but used differently located 2nd targets; each target pair is labeled with an italicized number and letter by its 2 nd target. Right: examples of saccadic sequences generated in response to each configuration. The sequences are from before inactivation only, so as to illustrate the normal baseline behavior. Mean 1st and 2nd saccade vectors are shown (as in Fig. 3C), although the 1st saccade vectors on each side mostly overlap. Horizontal and vertical SDs of all 2nd saccade end points are also shown. ---, vertical meridians. $B$ : single-step task configurations. In each trial, monkeys made a single saccade from a central fixation spot $(\bullet)$ to a target $(0)$.

and Fig. 5, $C$ and $D$, shows examples from configuration $C$. In all of these cases, the second saccade end points shifted significantly in the contraversive direction. As was illustrated in Fig. $2 C$ (middle), this implied that the underlying corollary discharge signals shrank. For comparison, changes in second saccade end points typically did not occur in trials involving ipsiversive first saccades (Fig. 5E) or in saline control injections (Fig. 5F). 
A
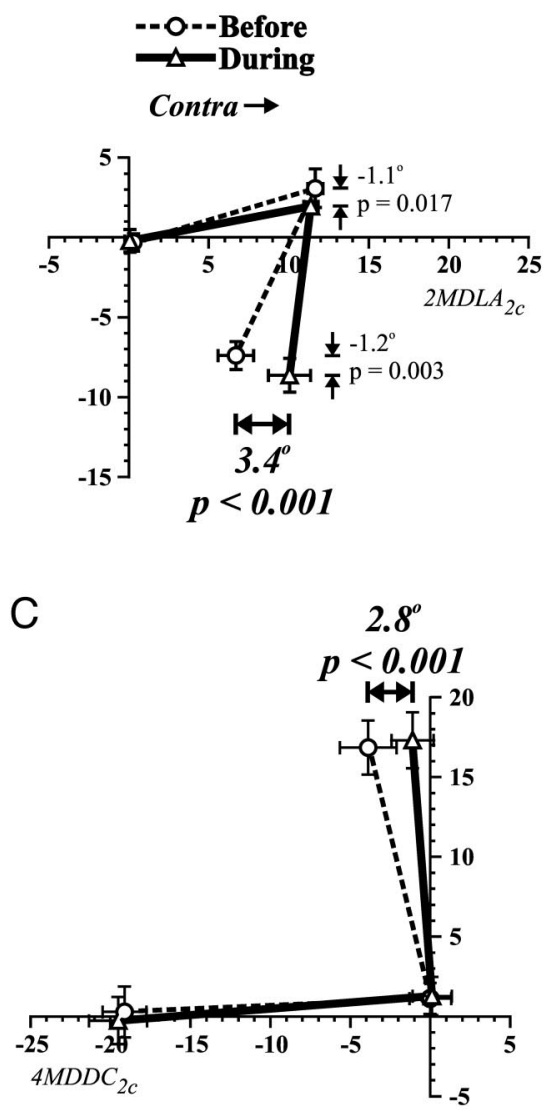

E

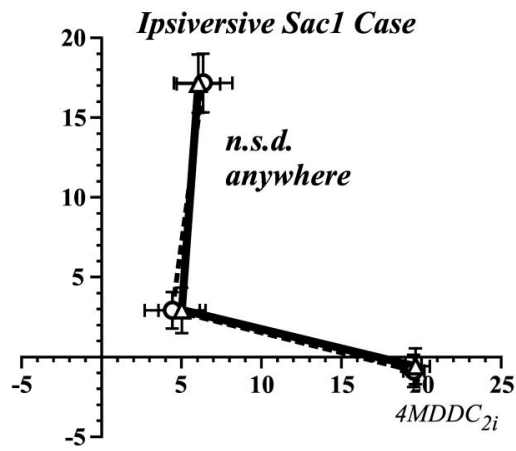

B
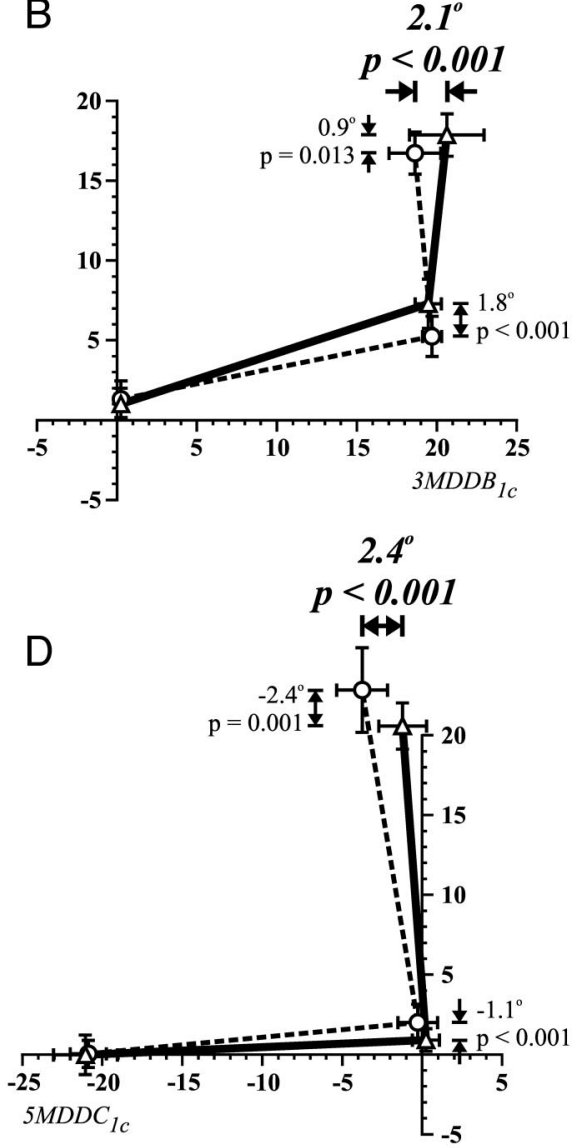

$\mathrm{F}$

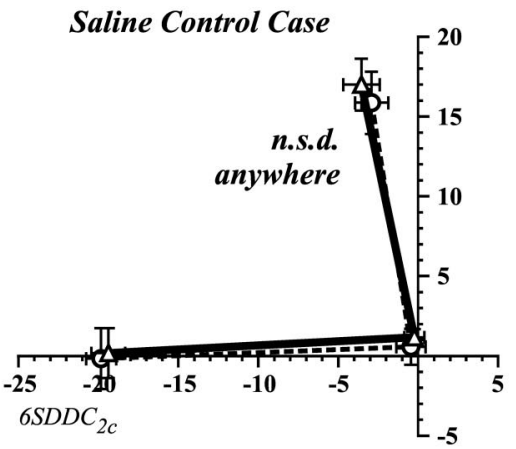

FIG. 5. Examples of impairments using various target configurations $(A-D)$ and of lack of impairments in ipsiversive $(E)$ and saline control $(F)$ trials. Note that the examples in $A-D$ are from 4 different inactivation experiments and that the data in $E$ and $F$ are the ipsiversive and saline control complements of the data shown in $C$. Occasionally, as in $A, B$, and $D$, contraversive shifts in 2 nd saccade end points were accompanied by vertical shifts, but these always seemed to be carried over from vertical shifts in 1 st saccade end points.

\section{Accuracy deficits}

Our first observation as shown in the preceding examples was that MD inactivation often caused a systematic shift in second saccade end points indicative of a corollary discharge accuracy deficit. Next we will document this effect quantitatively.

TRIALS INVOLVING CONTRAVERSIVE FIRST SACCADES. Figure 6 summarizes the results by pooling data from all injections and both monkeys. In each experiment, the data from a single target pair represented one "case" for analysis; e.g., one case was shown in Fig. $3 C$ and six more in Fig. 5, $A-F$. Combining all the inactivation experiments, there were 22 cases involving contraversive first saccades. In nearly every case $(82 \%, 18 / 22)$, there was a contraversive shift in second saccade end points during MD inactivation (Fig. 6A, left). Shifts were individually significant in half of the cases $(11 / 22)$ and the average shift was significantly greater than zero. The shifts were approximately the same in each monkey (Fig. 6A, left, inset graphs) and they occurred regardless of whether the task was performed in total darkness or in dim light (not shown; mean shifts were $1.12^{\circ}$ in $n=16$ dark cases and $1.13^{\circ}$ in $n=6$ dim light cases).

No other systematic shifts were seen. Along the vertical dimension (Fig. 6A, right), shifts were rare, significant ones were equally likely to be upward as downward, and the mean shift was not different from zero. End points of first saccades (Fig. 6B) were not shifted significantly in the horizontal direction during inactivation, except for a single case, and similarly were not shifted vertically on average (a few individual cases were, but they were as likely to be shifted upward as downward). Initial fixation locations (Fig. 6C) were unchanged during inactivation in both directions. 

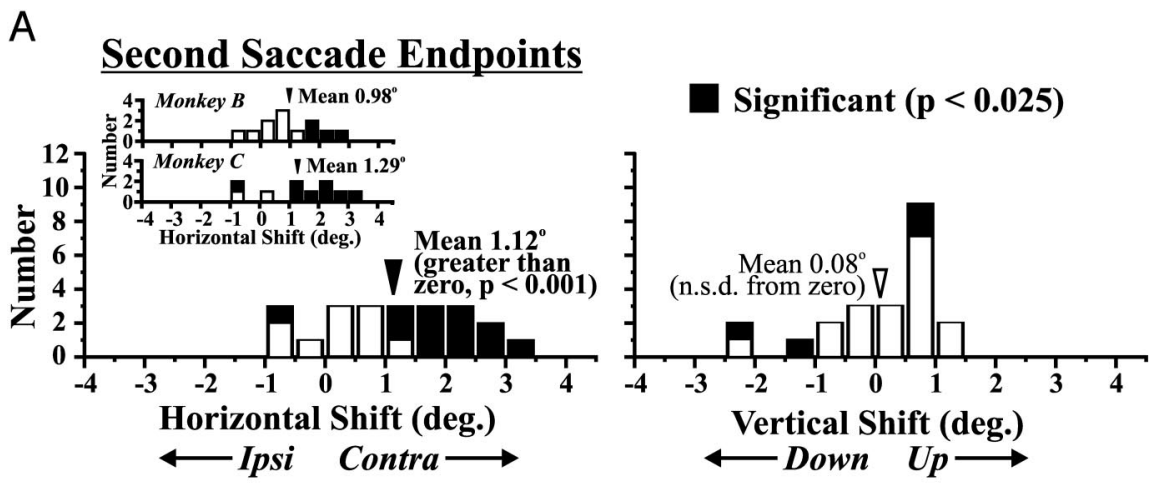

B

First Saccade Endpoints
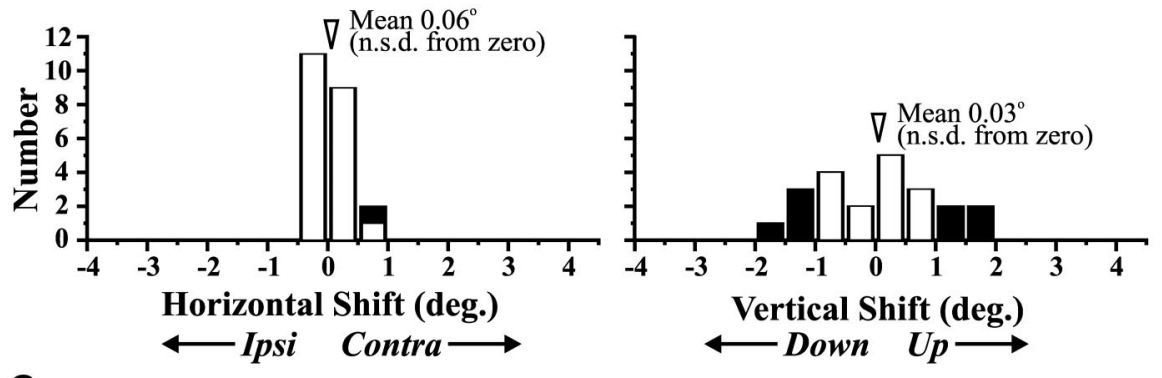

C

\section{Initial Fixations}
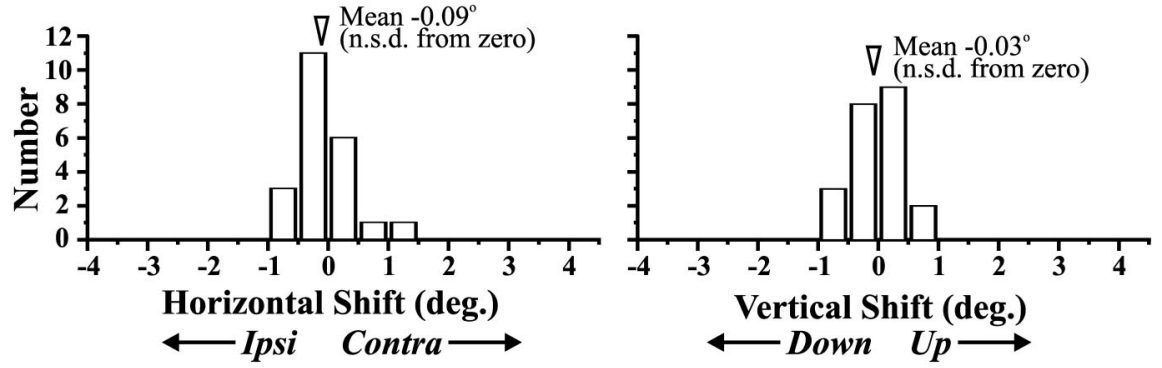

FIG. 6. Summary of data that revealed an accuracy deficit in corollary discharge. A: horizontal (left) and vertical (right) shifts of 2 nd saccade end points during inactivation relative to before. Insets: the data from the main graph are split into the component data from each monkey (the mean shift for each monkey was significantly greater than $0, P<0.025)$. Also shown are horizontal and vertical shifts in the 1st saccade end points $(B)$ and in initial fixations $(C)$ as well as the horizontal shifts of 2 nd saccade end points from trials involving ipsiversive 1st saccades $(D$, left $)$ and saline control trials (right). Some of these data were published previously (Sommer and Wurtz 2002).

\section{Second Saccade Endpoints in Comparison Cases}

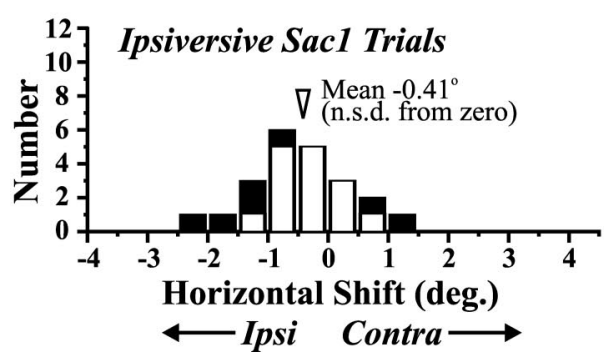

As noted in the preceding text, the contraversive direction of the shifts implied that the underlying corollary discharge vectors shrank (Fig. 2C, middle). It follows that the maximum possible contraversive shift should equal the first saccade amplitude $\left(10^{\circ}\right.$ in target configuration $A, 20^{\circ}$ in configurations $B$ and $C$ ). To estimate the severity of the deficit, we compared the observed shifts to these maximal shifts. As reported previously (Sommer and Wurtz 2002), in the 11 cases showing a significant shift (Fig. 6A, left), there was a $19 \%$ average deficit. Considering all 22 cases, there was a $10 \%$ average deficit.

IPSIVERSIVE SACCADES AND SALINE CONTROLS. We analyzed two other types of trials for comparison with the preceding results. First, because MD relay neurons represent contraversive saccades almost exclusively (Sommer and Wurtz 2004b), we expected MD inactivation to have little or no effect on corollary discharge of ipsiversive saccades. Second saccades following ipsiversive first saccades, therefore, should be unperturbed during MD inactivation, and that is what we found. Figure $6 D$, left, shows the distribution of horizontal shifts in second saccade end points for all 22 ipsiversive control cases; here, a corollary discharge deficit would be indicated by an ipsiversive shift. There were a few more individually significant ipsiversive shifts than contraversive ones (5 vs. 2) and the overall mean trended toward the ipsiversive direction, but this 
was not significant $(P>0.025)$. As a second point of comparison we expected that trials involving contraversive first saccades should be unaffected if only saline, sans muscimol, was injected; this too was found to be true (Fig. 6D, right).

ANGLE OF THE SHIFT IN SECOND SACCADE END POINTS. The average shift during inactivation, in trials involving contraversive first saccades, was significant horizontally but not vertically (Fig. 6A, left), but that does not necessarily mean that the exact angle of the shift was horizontal. If the shift angle were appreciably different from horizontal, it would imply that the underlying corollary discharge signal not only shrank on average but also rotated. To quantify the exact shift angle, in each case we connected the means of the second saccade end points before and during inactivation with a line. The angle of the line relative to horizontal (defined as $\phi$ ) was the shift angle while the length of the line (defined as $D$ ) was the shift distance. For the case shown in Fig. $3 C$, for example, the line had an angle $\phi=+25.9^{\circ}$ and a length $D=2.3^{\circ}$. We treated each line as a vector and plotted all of them in Fig. 7. Vectors from inactivation experiments in which the first saccade was contraversive are shown in Fig. $7 A$ (the vector corresponding to the Fig. $3 C$ example is shown with a dashed arrow). The overall mean vector (larger, white arrow) was highly significant $(P<0.01)$, as zero fell outside the $99 \%$ confidence ellipse regarding its tip location. This mean vector had $\phi=+5.0^{\circ}$ and $D=1.1^{\circ}$. Because the average shift was nearly horizontal, the underlying corollary discharge signal did seem to be shortened but not appreciably rotated. In contrast, for the cases involving ipsiversive first saccades (Fig. 7B) and for the saline controls (Fig. $7 C$ ), the mean vectors were not significant (the $95 \%$ confidence ellipses included 0), which confirms that these cases exhibited no overall shift.

\section{Precision deficits}

We expected that a precision deficit in corollary discharge would increase the scatter of second saccade end points (Fig. $2 C$, right), but this did not occur (Fig. 8A); in trials involving contraversive first saccades, the SD of second saccade end points did not change during inactivation (paired $t$-tests, $P=$ 0.36 for horizontal SD, 0.62 for vertical). If there was increased scatter, it was not detectable against the background scatter. A subtle deficit was suggested, however, by the fact that in Fig. $8 \mathrm{~A}$ more data points fell above the unity line than below it ( 25 vs. 19). This prompted us to try a different technique for identifying a precision deficit.

Our new approach was to perform a trial-by-trial analysis. We took advantage of the fact that, like all saccades, first saccades in the double-step task vary slightly from trial to trial. If corollary discharge is precise, it will change on each trial to constantly match the first saccade. To appreciate this, consider again the sharp-shooting analogy (Fig. 2B). Now, however, the shooter must hit a target that moves in a random direction before each shot. One can maintain the same accuracy as with a stable target by just aiming at the center of the "cloud" of target locations. The average of the shots will still be at the bull's-eye, but the scatter will increase; precision will suffer. To maintain good precision, one would have to match each target movement with a corresponding change in aim. Only this will keep the resulting shot cluster tight. Similarly, corol- lary discharge will be precise only if it changes on each trial to match the slightly varying saccade.

To measure how well corollary discharge matches the first saccade on a trial-by-trial basis, once again we used second saccades as our behavioral indicators. Figure $8 B$ shows one case of saccadic sequences during and before inactivation, and $C$ shows each individual second saccade vector. To see if the monkey detected where each second saccade started (i.e., where each 1st saccade landed), we replotted all the second saccades in Fig. $8 D$ by spreading them out along the vertical dimension while maintaining their relative horizontal locations (they are arranged so that the saccade starting most to the left is at top and that starting most to the right is at bottom-not chronologically by trial number). At least before inactivation (Fig. 8D, left), the monkey did act as if it knew where each second saccade began; moving from left to right, the vectors tend to swing counterclockwise as if compensating for the different initial positions. In contrast, during inactivation (Fig. $8 D$, right), the monkey showed little evidence of knowing where each first saccade landed; all the angles of the second saccades were about the same regardless of where the saccades began.

To quantify this effect, we compared the observed second saccade vectors (Fig. $8 C$ ) with the ideal vectors that would have been expected from perfect compensation. Ideal vectors were modeled (Fig. 9A) by connecting the initial position of each observed second saccade to the mean end-point location of the second saccades (we assume this mean location was where the monkey intended its saccades to land). For quantification, we measured saccadic direction $(\theta)$ and amplitude $(\rho$; see Fig. 8D). First we evaluated how well the monkey adjusted its second saccade directions. Figure $9 B$ plots observed $\theta$ versus ideal $\theta$ for every trial. Before inactivation (bold circles and line), the values were highly correlated with a linear regression slope near 1.0. Thus the monkey was quite adept at adjusting its second saccade directions, implying that it knew where each first saccade landed; corollary discharge was precise. During inactivation (thin triangles and line), however, precision seemed impaired because the correlation became insignificant. Next we evaluated how well the monkey adjusted its second saccade amplitudes. Figure $9 C$ shows that the monkey was poor at this both before and during inactivation (observed $\rho$ was not correlated with ideal $\rho$ ). The criterion level for a significant correlation was $P<0.025$ because we looked for changes in both direction and amplitude of the saccades.

The results of this example were typical, and Table 2 lists all the data. In trials involving contraversive first saccades, significant decreases during inactivation occurred in the percent of cases having a significant correlation between observed and ideal $\theta$ (Table 2, 1st row from top) and also in the average slope of the regression between observed and ideal $\theta$ (Table 2 , 2nd row). Figure $9 D$ (left) graphs this change in slope and, for comparison, the insignificant changes for ipsiversive first saccade trials (middle) and saline control trials (right). No other significant effects were found (Table 2).

The precision deficit represented by the regression slope data in Fig. 9D (left) was large but not total; it was a $60 \%$ deficit measured as a percentage of baseline ([0.97-0.39]/0.97). Average slopes decreased a similar amount in both monkeys (by 0.55 for monkey $B$, from 0.81 to 0.26 ; by 0.60 for monkey $C$, from 1.16 to 0.56 ) and in both ambient light conditions (by 

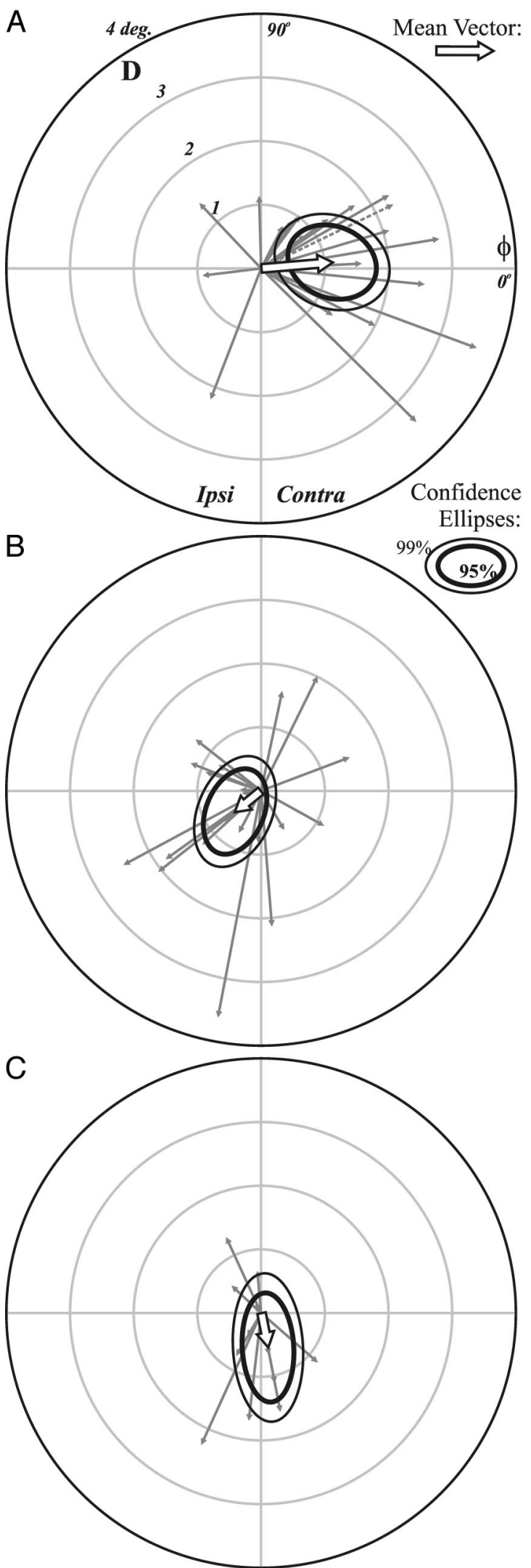

FIG. 7. Exact directions of 2nd saccade end-point shifts. Plotted are the vectors representing the shift in each individual case, the mean vectors, an confidence ellipses regarding the mean vector tips, for trials involving contraversive 1st saccades $(A)$, trials involving ipsiversive 1st saccades $(B)$, and saline control trials $(C)$
A
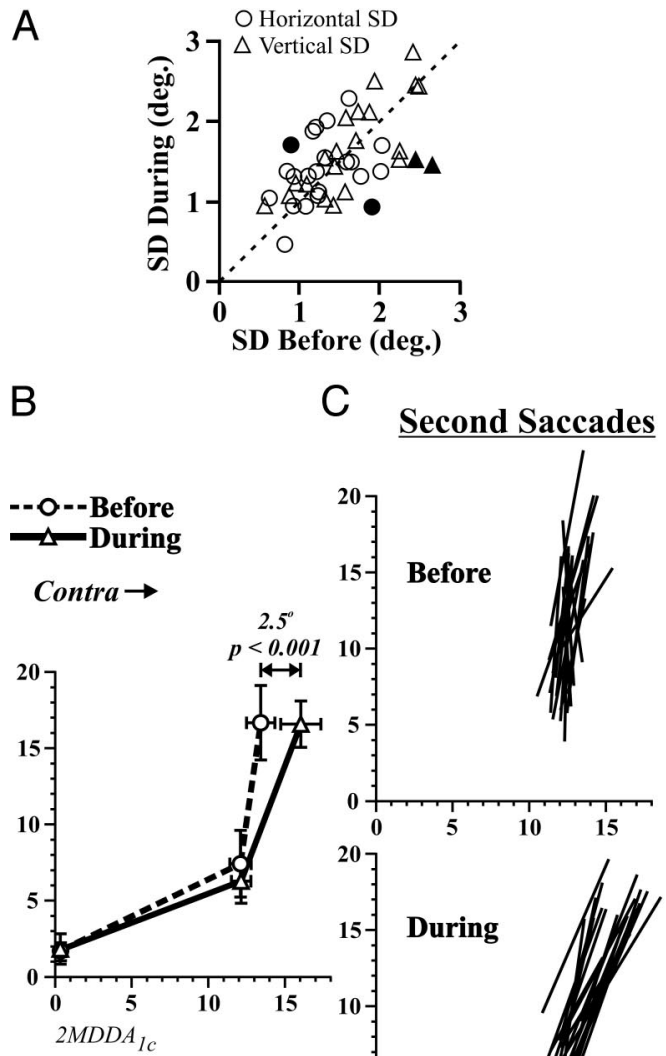

Before

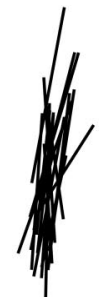

$\mathrm{D}$

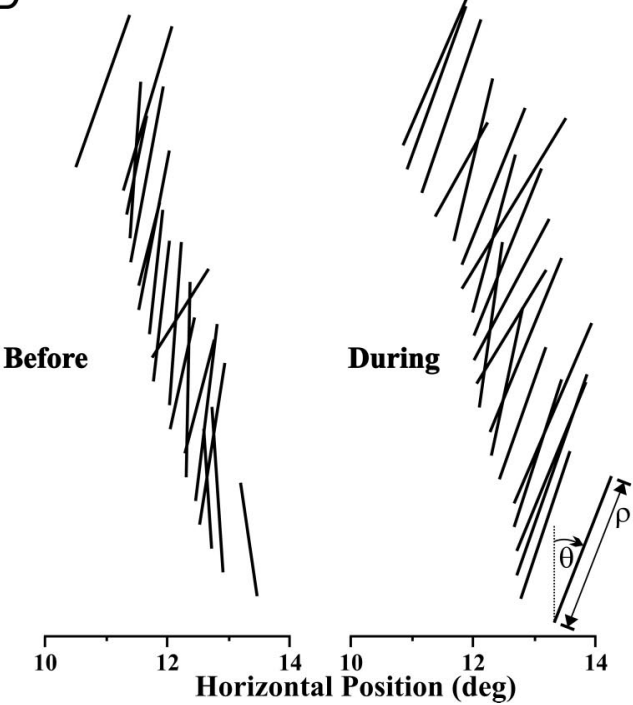

FIG. 8. Analysis of corollary discharge precision. A: the SDs of second saccade end points during vs. before MD inactivation. $\bullet$ and $\boldsymbol{\Delta}$ show cases in which the SD changed significantly $(P<0.025)$ during inactivation. $B$ : summary data from an example injection. $C$ : the individual second saccades from this example before (top) and during (bottom) inactivation. We depicted each saccade as a vector by connecting its initial and final position with a line. $D$ : same saccades as in $C$ but spread along the vertical dimension so that each can be seen distinctly. Each $x$ axis is expanded as compared with that in $C$. At the lower right are illustrated our 2 quantifications of each saccade: the saccadic direction $(\theta)$ and amplitude $(\rho)$. A was modified from Sommer and Wurtz (2002). 

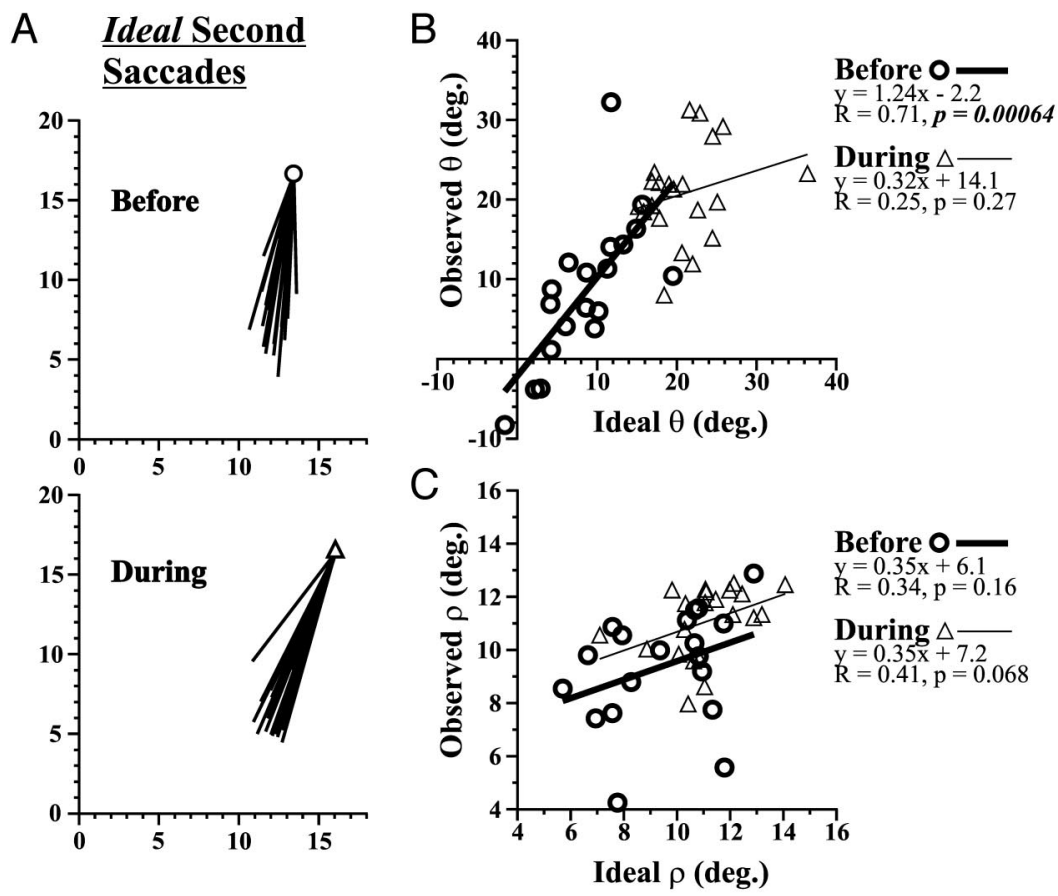

Overall Results, All Injections

Before .....

Curing -

Contraversive Sac1

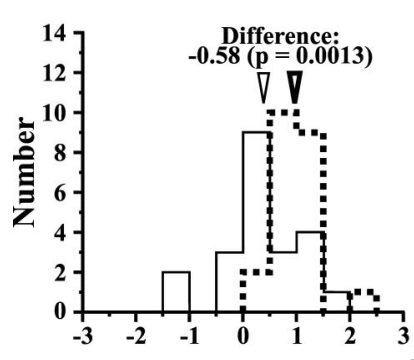

Ipsiversive Sac1 -0.12 (n.s.d.)

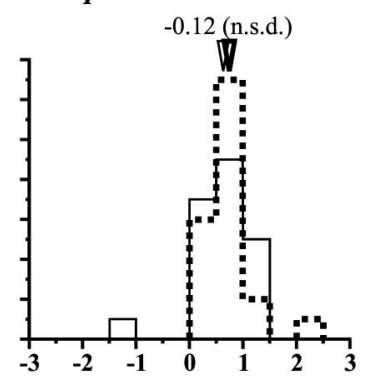

Slope (Observed $\theta$ vs. Ideal $\theta$ )
Saline Controls

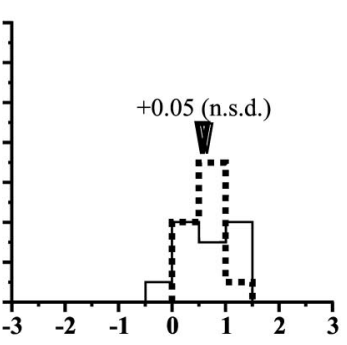

FIG. 9. Comparison of observed 2nd saccade vectors with ideal ones expected if corollary discharge had been perfectly precise. $A$ : ideally compensating 2 nd saccades for the Fig. 8 example case (cf. Fig. $8 C$ ). Circle and triangle show means of the 2 nd saccade end points before and during inactivation, respectively (from Fig. $8 B) . B$ : analysis of how well the monkey adjusted its 2 nd saccade directions to account for trial-by-trial fluctuations in its 1 st saccades. The observed $\theta$ for each 2 nd saccade (from the vectors in Fig. 8, $C$ or $D$ ) is plotted against the ideal $\theta$ (from $A$ of this figure). Before inactivation there was a direct correlation between observed $\theta$ and ideal $\theta$ with a linear regression slope near unity (1.24), but during inactivation the correlation was not significant and the slope was only 0.32. $C$ : same as in $B$ except now the ability to adjust 2 nd saccade amplitude trial-by-trial is evaluated; observed $\rho$ is plotted vs. ideal $\rho$. Neither correlation is significant, either before or during inactivation. $D$ : overall results showing how the ability to adjust the direction of the 2nd saccade trialby-trial was impaired by MD inactivation. For trials involving contraversive 1st saccades (left), the average slope of the linear regression describing the observed $\theta$ vs. ideal $\theta$ relationship dropped by 0.58 , which was highly significant as shown. In contrast, for trials involving ipsiversive 1st saccades (middle) and for saline control trials (right), there was no significant change in slope.
0.50 for dim light, from 1.16 to 0.66 ; by 0.60 for darkness, from 0.89 to 0.29 ). Finally, the precision deficit appeared to be more pervasive than the accuracy deficit. Precision deficits occurred both in the 11 cases that had an accuracy deficit (i.e., the 11 cases exhibiting an individually significant contraversive shift in second saccade end points-see Fig. 6A, left), with average slopes decreasing by 0.54 , from 0.82 to 0.28 , and in the other 11 cases with no accuracy deficit (average slopes decreased by 0.61 , from 1.12 to 0.51 ). The importance of this is questionable, however; it could just be that the precision analysis was more sensitive than the accuracy analysis.

As an aside, these results also demonstrate that the monkeys did not preprogram their saccadic sequences. The monkeys normally tailored the direction of their second saccades to compensate for changes in their first saccade end points, meaning that they only made a second saccade after learning about the first one. Our strategies of not over-training the monkeys and frequently changing the target configurations therefore seemed to successfully prevent preprogramming.

In sum, the precision analysis revealed both a positive and negative result. The positive finding was twofold: monkeys normally adjusted their second saccade directions from trial to trial to compensate for slight fluctuations in first saccades, and this was disrupted by inactivation. This shows that corollary discharge is normally precise and that MD inactivation impairs this precision. The negative finding was that monkeys showed little ability to adjust their second saccade amplitudes. This may explain why we found no precision deficit in our initial SD analysis (Fig. 8A). Second saccade amplitudes both before and during inactivation were nonideal-essentially "noisy"-and this may have contributed so much scatter to the end points that SD increases caused by an impaired ability to adjust second saccade directions could not be detected. Only by analyzing second saccade directions in isolation could we uncover the precision deficit.

\section{Generation of single saccades}

In the preceding sections, we considered whether corollary discharge, i.e., information about saccades, was impaired by 
TABLE 2. Results of precision analysis

\begin{tabular}{|c|c|c|c|}
\hline \multirow[b]{2}{*}{ Change During vs. Before Inactivation } & \multicolumn{2}{|c|}{ Sac1 Cases } & \multirow[b]{2}{*}{ Saline Control Cases } \\
\hline & Contraversive & Ipsiversive & \\
\hline \multicolumn{4}{|l|}{ Second saccade direction: actual $\theta$ vs. ideal $\theta$} \\
\hline Percent correlated* & $\begin{array}{c}-36 \% \\
(50 \% ; 14 \% ; 0.022)\end{array}$ & $\begin{array}{c}-14 \% \\
(59 \% ; 45 \% ; 0.55)\end{array}$ & $\begin{array}{c}8 \% \\
(17 \% ; 25 \% ; 1.0)\end{array}$ \\
\hline Regression slope $\dagger$ & $\begin{array}{c}-0.58 \\
(0.97 ; 0.39 ; 0.0013)\end{array}$ & $\begin{array}{c}-0.12 \\
(0.75 ; 0.63 ; 0.43)\end{array}$ & $\begin{array}{c}0.06 \\
(0.57 ; 0.62 ; 0.65)\end{array}$ \\
\hline Regression intercept & $\begin{array}{c}-3.6 \\
(-1.7 ;-5.2 ; 0.49)\end{array}$ & $\begin{array}{c}-0.04 \\
(-7.0 ;-7.0 ; 0.99)\end{array}$ & $\begin{array}{c}-0.31 \\
(-2.4 ;-2.7 ; 0.83)\end{array}$ \\
\hline \multicolumn{4}{|l|}{ Second saccade amplitude: actual $\rho$ vs. ideal $\rho$} \\
\hline Percent correlated & $\begin{array}{c}-18 \% \\
(36 \% ; 18 \% ; 0.31)\end{array}$ & $\begin{array}{c}-9 \% \\
(50 \% ; 41 \% ; 0.76)\end{array}$ & $\begin{array}{c}0 \% \\
(18 \% ; 18 \% ; 1.0)\end{array}$ \\
\hline Regression slope & $\begin{array}{c}-0.46 \\
(0.74 ; 0.28 ; 0.028)\end{array}$ & $\begin{array}{c}-0.14 \\
(0.69 ; 0.54 ; 0.38)\end{array}$ & $\begin{array}{c}0.19 \\
(0.59 ; 0.79 ; 0.47)\end{array}$ \\
\hline Regression intercept & $\begin{array}{c}5.1 \\
(3.4 ; 8.5 ; 0.079)\end{array}$ & $\begin{array}{c}2.2 \\
(4.7 ; 7.0 ; 0.32)\end{array}$ & $\begin{array}{c}-4.6 \\
(8.2 ; 3.6 ; 0.22)\end{array}$ \\
\hline
\end{tabular}

\footnotetext{
* Percent correlated cell contents show difference in the percent of cases having a significant correlation during vs. before inactivation (parenthetical data:

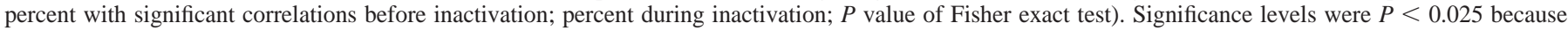

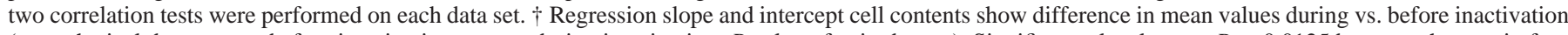

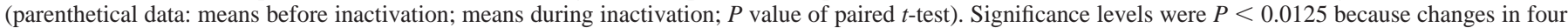

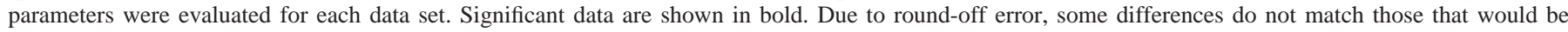
calculated from the parenthesized means.
}

MD inactivation; next we consider whether saccade generation itself was impaired. In brief, it was not. As shown in the preceding text, inactivation had little or no effect on the generation of contraversive first saccades in the double-step task (Fig. 6B), and previously (Sommer and Wurtz 2002) we showed one example of unaffected saccades made in the single-step task, demonstrated that the dynamics of single saccades were unaffected, and reported that there were no overall accuracy or latency deficits. Here we illustrate saccades made in a different version of the single-step task and provide complete accuracy, precision, and latency results.

Figure $10 A$ shows an example experiment that was notable because it contained the largest changes in single saccades during MD inactivation that we ever found. In contrast to the previously published example that showed saccades from the target-present version of the task (Sommer and Wurtz 2002), this example (Fig. 10A) shows saccades from the target-absent version. The target-absent version was more challenging in that the target was flashed only briefly and its location had to be remembered during the reaction time (see METHODS). In each trial, the monkey made a single saccade from the center to a target appearing at one of the locations shown in Fig. $4 B$ (configuration $B$ ), and shown are the means and SDs of the saccadic end points before and during MD inactivation along with the radial distance ( $D$ value) of each shift. To see if the shifts were significant, we tested them along the two cardinal axes, as we did in the double-step task, and superscripts following the $D$ values indicate end points that were significantly shifted horizontally (h) or vertically (v). As can be seen, shifts were small in magnitude, primarily vertical in direction, and about as likely to affect contraversive as ipsiversive saccades. Figure $10 B$ shows the distributions of shift sizes of contraversive saccades (top) and ipsiversive saccades (bottom) from all the single-step cases. The average shift size was not significantly different between contraversive and ipsiversive saccades, and a few individually significant horizontal or vertical shifts were seen for both contraversive and ipsiversive saccades. The precision of single saccades was not affected by inactivation either (Fig. $10 C$; all paired $t$-tests of during-before changes yielded $P>0.025)$. Unlike in the double-step task, we saw no alternative way of testing for a precision deficit; it is possible there was a small deficit in the precision of saccade generation that was undetectable. Finally, reaction times were not affected (Fig. 10D; all paired $t$-tests yielded $P>0.05)$.

In sum, taking these single-step task results together with the lack of effect on first saccades of the double-step task, we conclude that MD inactivation has no effect on the ability to generate saccades. It should be noted that, as a point of comparison, similar injections of muscimol cause severe, lateralized impairments of saccade generation in the SC (Aizawa and Wurtz 1998; Hikosaka and Wurtz 1985) and in the FEF (Dias and Segraves 1999; Sommer and Tehovnik 1997), presumably due to silencing these structures' descending output neurons (Everling and Munoz 2000; Munoz et al. 1991; Segraves 1992; Segraves and Goldberg 1987; Sommer and Wurtz 2000, 2001).

\section{Alternative explanations}

Returning to the double-step task results, we think that corollary discharge deficits provide the simplest explanation for the second saccade disruptions in trials involving contraversive first saccades. Nevertheless, alternative explanations should be considered.

WERE SECOND SACCADE END-POINT SHIFTS DUE TO FIRST SACCADE END-POINT SHIFTS? Figure $6 B$ (left) showed that in one case there was a significant contraversive shift in first saccade end points during MD inactivation, and Fig. 11A (left) shows the average saccades from this case. The slight $\left(0.8^{\circ}\right)$ but significant shift in first saccade end points may have contributed to the significant shift in second saccade end points of $1.8^{\circ}$. To see if there was an accumulation of horizontal error from first to second saccades in general, we plotted the horizontal shift in second saccade end points as a function of the horizontal shift in first saccade end points (Fig. 11A, right). If the errors accumulated, then larger first saccade shifts should 
A Single Saccades: Example

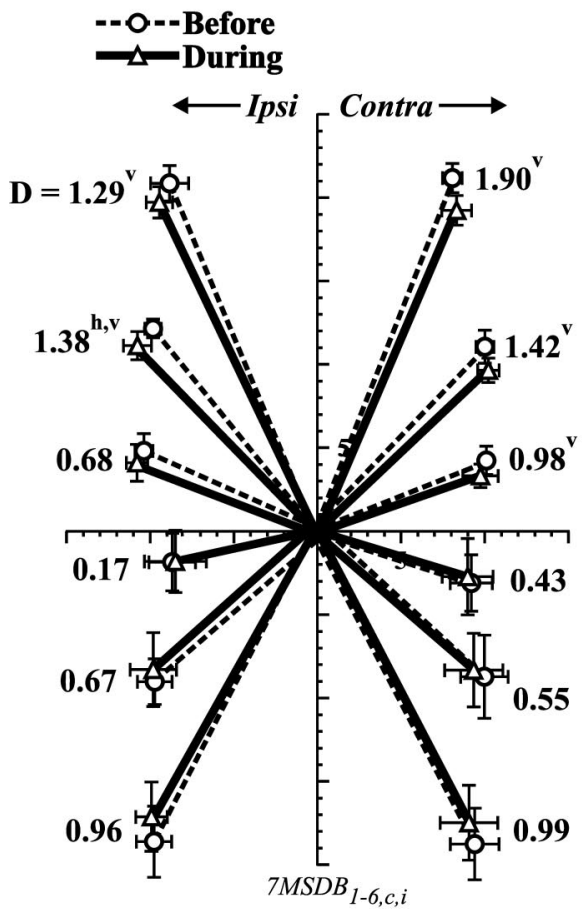

B

Accuracy

Significant shift either horizontally

or vertically $(p<0.025)$

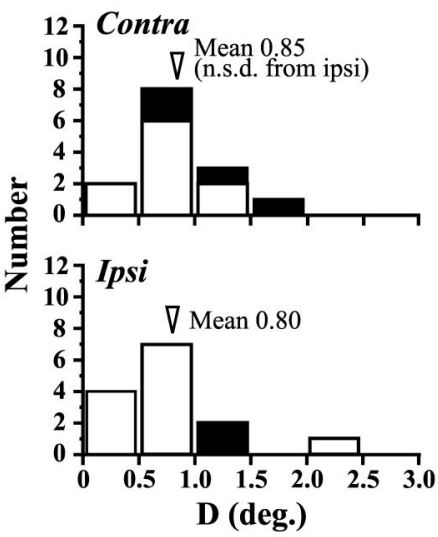

FIG. 10. Lack of impairments to saccades made in the single-step task. See RESULTS for details. A: summary of single saccades made before and during 1 example inactivation. $B$ : histograms showing how accuracy was affected by inactivation. $C$ : scatter plots of the SDs of saccadic end-point clusters before versus during inactivation as a measure of how precision was affected by inactivation. $D$ : scatter plots of reaction times of the saccades before vs. during inactivation. The data in $B-D$ represent 14 pairs of before-during saccadic end points in each direction (contraversive and ipsiversive), 12 of which were from the target-absent version of the task and 2 from the target-present version; we collected some end-point pairs that we later omitted from analysis due to low number of saccades to analyze (we required a minimum of 5 in each before or during end-point cluster) or because they were vertically directed (see Fig. $4 B$, configuration $A$ ) rather than ipsi- or contraversive.
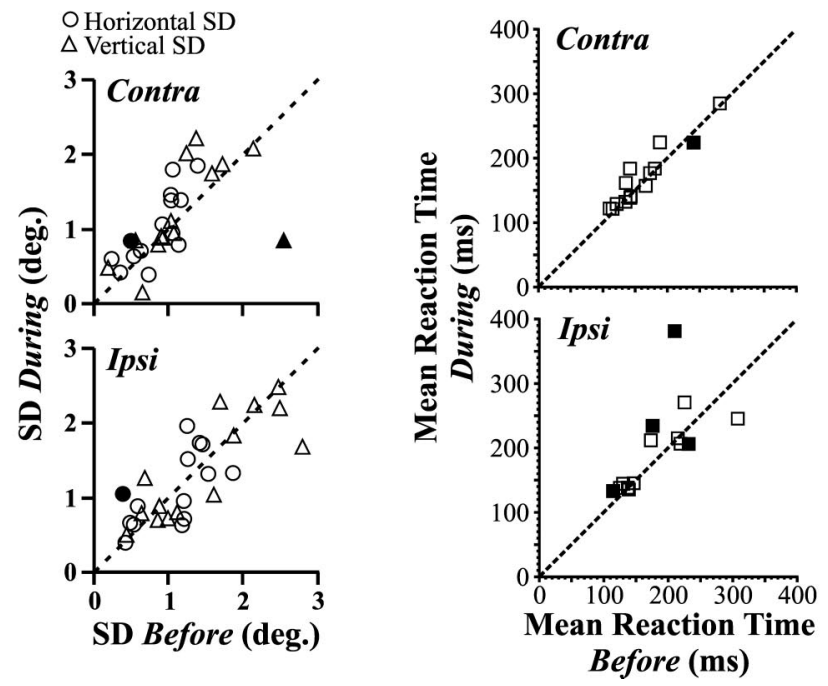

have tended to produce larger second saccade shifts; the data should be directly correlated. There was no correlation, however $(P=0.888)$. First saccade end-point shifts did not seem to cause second saccade end-point shifts.

WERE THE DEFICITS ARTIFACTS OF CUMULATIVE DAMAGE? It could also be that the behavior just kept worsening every day due to progressive neuronal damage so that changes during versus before inactivation were artifacts of collecting the data on sequential days. Arguing against this, we found no evidence for cumulative damage; deficits always recovered after inactivation. Figure $11 B$ (left) illustrates one example. During inactivation the second saccade end points shifted contraversively, but after inactivation, i.e., the next day, the second saccade end

points were not significantly different from those collected before the inactivation. The behavior had completely recovered. This was true for all 11 cases in which there was a significant contraversive shift during inactivation (Fig. 11B, middle); in no case was there still a significant contraversive shift after the inactivation, and the overall mean was not different from zero. Precision deficits also recovered in these 11 cases. As noted in the preceding text, the regression slope between observed $\theta$ and ideal $\theta$ for these cases decreased from an average of 0.82 before inactivation to 0.28 during inactivation, but after inactivation the average slope rose to 0.81 , representing almost perfect recovery (Fig. 11B, right). 

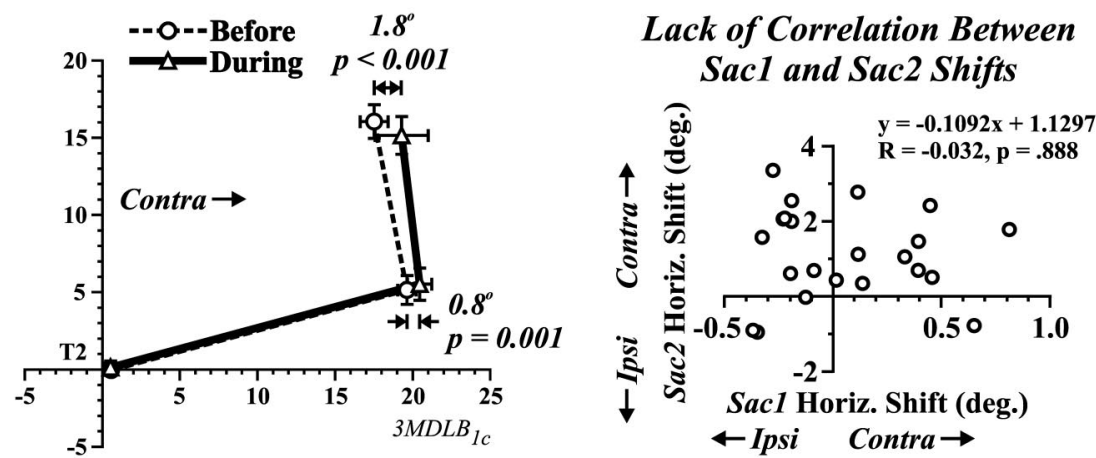

\section{B Were Sac2 shifts due to cumulative tissue damage?}
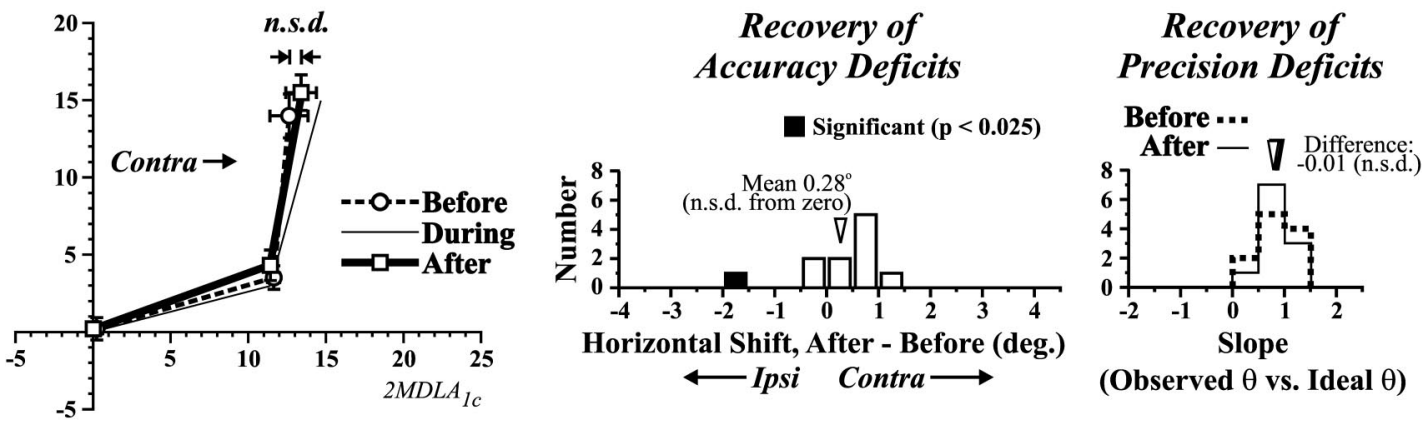

FIG. 11. First 2 possible alternative explanations for the double-step accuracy deficit. $A$ : maybe the shifts in 2 nd saccades were due to horizontal errors of 1st saccades. Left: the single case in which there was a significant contraversive shift in 1 st saccade end points. Right: plot of the horizontal shift in 2 nd saccade end points for all cases (ordinate) as a function of the horizontal shift in 1 st saccade end points. There was no relation; larger 1 st saccade shifts were not associated with larger 2 nd saccade shifts. Horizontal errors did not accumulate. $B$ : maybe the shifts in 2 nd saccades were due to accumulating neuronal damage. Left: an example showing "after" data compared with before data. For reference, the during data are shown too, using lines but no symbols. After data were not significantly different from before data; recovery was complete. Middle: for the 11 cases in which there was a significant accuracy deficit, the behavior recovered completely after inactivation. Right: also, for these 11 cases there was no lingering precision deficit. The after data were collected 1 day following inactivation in 8 of the 11 cases and 1-3 wk later in the 3 other cases.

WERE SECOND SACCADE END-POINT SHIFTS DUE TO GENERAL ROTATIONS OF ALL SACCADES? It could also be that the second saccade end-point shifts were an artifact of a direction deficit in saccade generation; maybe all saccades rotated contraversively. For example, we assumed that the second saccade end points in Fig. 12A (same as in Fig. 5C) shifted rightward because of inaccurate corollary discharge. It could be, however, that any saccade made in an obliquely upward direction would have rotated to the right. To test this, in two of the single-saccade control tasks discussed in the preceding text, we had targets arranged (configuration $B$ in Fig. $4 B$ ) so as to match the double-step target arrangement (configuration $C$ in Fig. 4A) during the same injections, and we asked whether single, obliquely upward saccades also were rotated. They were not. Figure $12 B$ shows the single saccade control that matched the trial type of $A$. The single saccades were much more accurate than the second saccades of the double-step task, of course, as would be expected. During inactivation, there was no rotation and consequently no contraversive shift (Fig. 12B, top); this was always the case (bottom).

WERE THE SHIFTS DUE TO WARPED VISUAL OR MEMORY REPRESENTATIONS? If visual perception or working memory were spatially perturbed, a monkey might think that the second target appeared more contralaterally than it actually did. This could be why second saccades were aimed further into contralateral space during inactivation. To test this, we again looked at control saccades made in the single-saccade task (Fig. 12C). These saccades actually originated from the center of the screen (see Fig. $4 B$, configuration $B$ ), but for ease of comparison with the double-step task (cf. Fig. 12A), we plot the data as if the saccades started $20^{\circ}$ to the left. For the visual system, the initial fixation location should be irrelevant as it is the retinal location that matters (we ignore any torsional difference between fixating centrally versus peripherally). The target timing and memory requirements in these target-absent single saccade trials were designed to match as closely as possible those of the second target in the double-step trials (see METHODS). There was little evidence for any sort of warping of visual or memory space (Fig. 12C). The example (Fig. 12C, top) shows the only significant contraversive shift in single saccade end points during inactivation that we found. On average, there was no significant shift (Fig. 12C, bottom).

WERE PRECISION DEFICITS DUE TO IMPRECISE VISION OR MEMORY? An alternative explanation for the precision deficits also posits a visual or memory problem. Perhaps corollary discharge precision was normal during inactivation, but the 


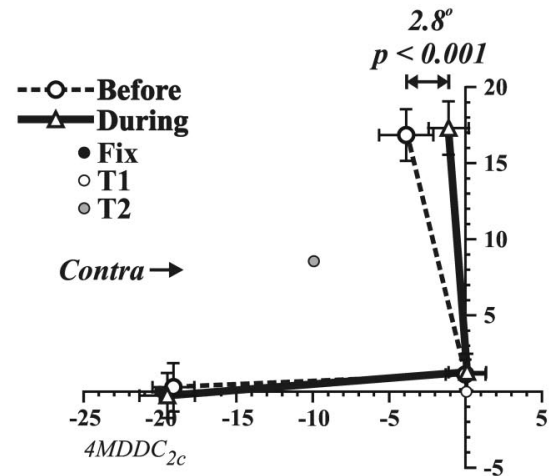

\section{B}

\section{Due to a rotation of all saccades?}
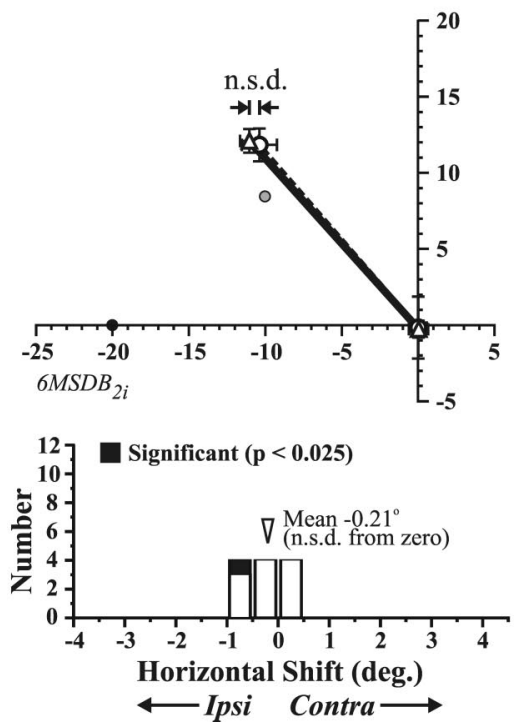

precision of detecting or remembering the target location was impaired; this also could have caused second saccade directions to deviate from the ideal during inactivation as in Fig. $9 B$. We found no evidence for this, however. Such impairments should have increased the scatter of second saccade end points, but this did not occur [Fig. 8A; testing for such a visual or memory deficit was in fact the original motivation for analyzing these data in Sommer and Wurtz (2002)]. As a follow-up, we looked at precision in the target-absent version of the single-step task. Detecting and remembering the target in this task should be of similar difficulty as detecting and remembering the second target in the double-step task. Precision in the single-step task, however, was unaffected by inactivation (Fig. 10C; nearly all the data are from the target-absent version). From what we can tell, therefore, the precision of detecting and remembering the second target remained normal. The best explanation for the deficit in compensation (Fig. $9 B$ and $D$, left ) is that the precision of corollary discharge was impaired.

\section{Bilateral impairments}

All the deficits in the double-step task discussed thus far affected only trials in which the first saccade was contraversive, but other, bilateral, impairments also occurred.
FIG. 12. Third and fourth possible alternative explanations: maybe all saccades were rotated or visual/memory space was warped. A: example case showing a contraversive shift in 2 nd saccade end points (from Fig. 5C). $B$ : single-step control case for this particular example showing that saccades did not just rotate clockwise to cause a contraversive shift (top) and that this was true in general (bottom). C: another single-step control case for this example, showing the only possible evidence for a contraversive warping of visual perception or memory (top). This case was the exception to the rule; in general (bottom) there were no shifts in these vision- and memorymatched control trials. 


\section{A Reaction Times $\square$ Before \\ - During}

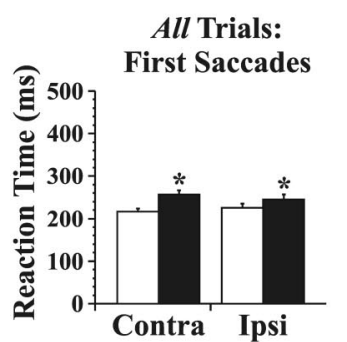

B Performance

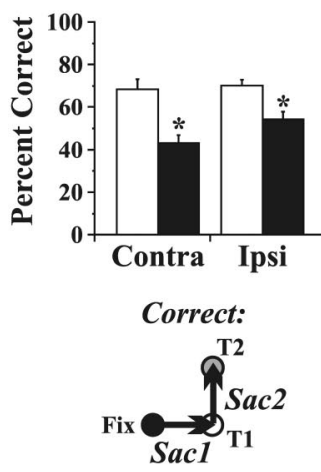

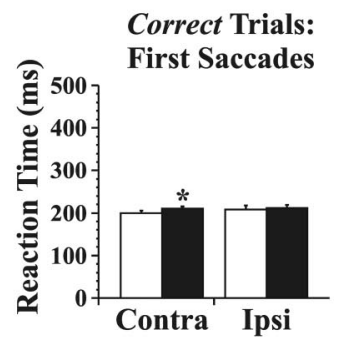
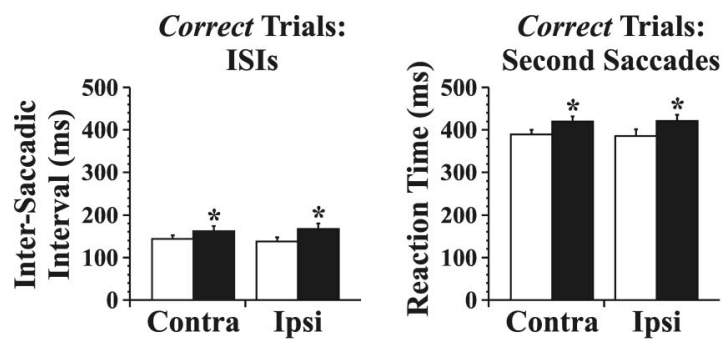

C Types of Errors
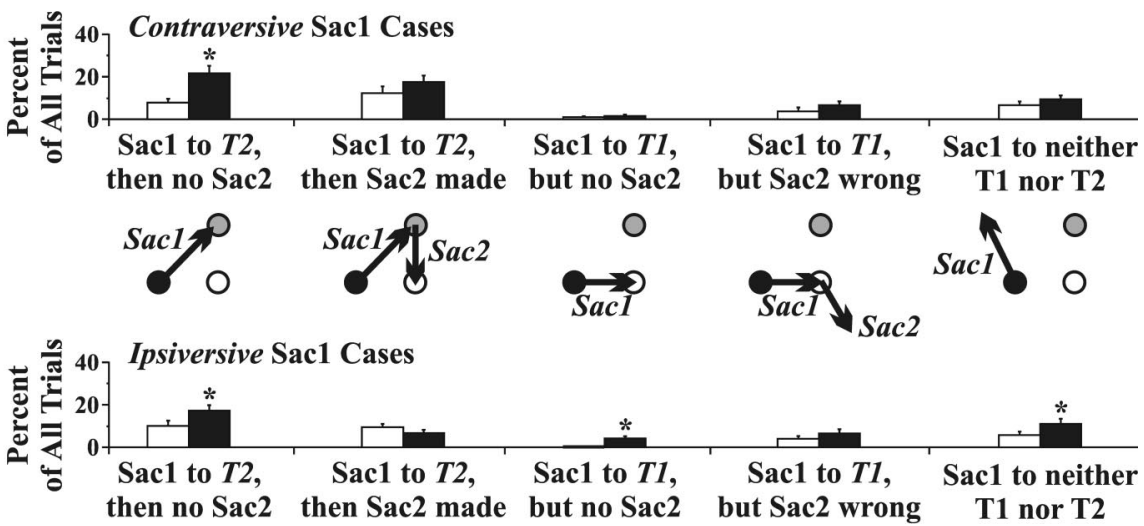

FIG. 13. Bilateral effects of MD inactivation. $A$ : increased reaction times. Bars show means + SEs of the average reaction times (or inter-saccadic intervals) of individual cases; each bar thus represents $n=22$ cases. Asterisks label significant changes (at $P<0.05$ ) during inactivation vs. before. From left to right are graphed the reaction times of 1st saccades from all trials and, from correct trials only, the reaction times of 1st saccades, the inter-saccadic intervals (ISIs), and the reaction times of 2nd saccades. All reaction times were measured relative to the appearance of the 1st target. $B$ : impaired overall performance. The bars show means + SEs of the average percent corrects from each case $(n=22$ cases per bar). A schematic depicting correct performance is shown below the bar graph. $C$ : distribution of the various types of error trials before and during inactivation from trials involving contraversive (top) and ipsiversive (bottom) 1st saccades. Underneath the top distribution, each class of error trial is diagrammed below its respective spot on the abscissa.

curred in trials involving ipsiversive first saccades (Fig. 13C, bottom): errors in which first saccades were correct but then no second saccades appeared (middle bars) and errors in which the first saccade headed to neither the first nor the second target (rightmost bars)

GENERAL LETHARGY. The monkeys occasionally seemed to become lethargic during inactivation. We were very familiar with the monkeys' behaviors, so when the following effects occurred less than an hour after muscimol injection (once with monkey $B$, twice with monkey $C$ ), we thought they were striking: the monkeys shut their eyes, ceased to work even for greater reward, and slept. When this occurred, we aborted the experiment and returned the monkeys to their cages. The next day the monkeys were completely recovered. None of the data in this report were collected during such episodes.

We do not know the underlying cause of the reaction time increases, the performance deficits, or the lethargy. Importantly, however, because all these impairments were bilateral, they could not have been related to the highly lateralized second saccade impairments that we considered markers of corollary discharge deficits.

\section{I S C U S S I O N}

The present results, taken together with the recording results of the accompanying paper (Sommer and Wurtz 2004b), pro- vide multiple lines of evidence supporting the hypothesis that the SC-MD-FEF pathway plays a role in corollary discharge. The accompanying paper showed that the pathway carries signals that start just prior to contraversive saccades and represent the approximate size and direction of the saccades. The presaccadic timing means that the activity is of central origin, not proprioceptive, the representation of saccadic vector means that information about specific upcoming movements is available, and the ascending route of the activity implies that it is an informative signal rather than a movement command. We hypothesized, therefore that the pathway conveys corollary discharge of contraversive saccades. To test this hypothesis, we inactivated MD relay neurons, thus interrupting signal flow in the pathway, while monkeys performed a double-step task that permitted inferences about covert corollary discharge deficits through examination of overt changes in second saccades. Mean second saccade end points were systematically shifted, implying a deficit in the accuracy of corollary discharge, and the ability to compensate for trial-by-trial fluctuations in first saccades was disrupted, implying a deficit in the precision of corollary discharge. These deficits occurred only in trials involving contraversive first saccades as predicted by the contraversive representation of saccades by MD relay neurons. Although information about saccades was impaired, saccade generation itself was not as shown by unaffected first saccades 
in the double-step task and single saccades in the single-step task. We think the simplest explanation for all these results, and that which binds together the results of our recording and inactivation studies most tightly, is that the SC-MD-FEF pathway conveys corollary discharge signals.

\section{Nature of the corollary discharge deficit}

SINGLE INACTIVATION DISRUPTS BOTH THE ACCURACY AND PRECISION OF COROLLARY DISCHARGE. Just like a sharpshooter might experience both accuracy and precision deficits from a single injury, say to the spinal cord, so might a monkey experience both accuracy and precision deficits from one insult to the overall corollary discharge representation in the brain. We think that is what happened when we inactivated MD: we deprived cortex of a critical source of information about saccades, and this single blow compromised both the accuracy and precision of a monkey's estimate as to where its saccades went.

To understand how the loss of input from the SC-MD-FEF pathway could cause both accuracy and precision deficits in corollary discharge, it is helpful to review the characteristics of this input. The putative corollary discharge signals, namely presaccadic discharges in the pathway, are high-frequency bursts that start just before the saccade and quit just after it (Sommer and Wurtz 2004b). By removing this strong, sharp activity, the brain's overall corollary discharge representation of the saccade might be dampened and broadened. Such a compromise in the signal-to-noise ratio should impair both the accuracy and precision of corollary discharge. Analogously, weaker saccadic commands in the SC seem to render saccade generation both less accurate and less precise (Edelman and Goldberg 2001; Stanford and Sparks 1994; Stanford et al. 1996).

WHY WERE THE DEFICITS PARTIAL? Although both accuracy and precision of corollary discharge were impaired, neither deficit was complete: accuracy was disrupted by $\sim 10-20 \%$ and precision by $60 \%$. We think the apparently different sizes of these deficits were unimportant as they may be attributable to the different analyses involved. The salient point is that MD inactivation did not totally eliminate corollary discharge. We see at least four possible explanations for this. First, the pathway might not have been completely silenced; our single-point injections may not have successfully inactivated all of the MD relay neurons. Second, corollary discharge signals might have continued to reach cortex through other transthalamic pathways. Many motor-related subcortical regions innervate thalamus (Steriade et al. 1997), and the dentate nucleus of the cerebellum and the substantia nigra, in particular, have been shown to contact relay neurons projecting to FEF (Lynch et al. 1994). Third, some corollary discharge signals might originate within cortex. The FEF, for example, sends saccadic instructions downstream and may simultaneously generate corollaries of those signals (Everling and Munoz 2000; Segraves and Goldberg 1987; Sommer and Wurtz 2000). Knocking out the SC-MD-FEF pathway would leave such intracortical signals unaffected. Fourth, even if corollary discharge had been totally lost, it is conceivable that monkeys relied to some extent on proprioceptive information as a "backup" way to perform the double-step task.

SPREAD OF INACTIVATION FROM MD. Our goal was to inactivate MD relay neurons, and we are convinced that we did this because we aimed muscimol directly at them. Neighboring neurons, however, were likely inactivated as well; we did not measure the spread of inactivation. There was probably a medial bias to the spread considering that the internal medullary lamina should limit diffusion laterally (similar sheets of fibers were found to be effective boundaries in other injection studies) (e.g., Weese et al. 1999). Nonetheless we recognize that our corollary discharge deficits may have been caused in part by inactivating important lateral thalamic areas, such as the intralaminar nuclei (Schlag-Rey and Schlag 1989), that may contain relay neurons of other ascending pathways. Evaluating this possibility would require one to physiologically identify relay neurons of the other pathways, aim inactivations directly at them, and compare any corollary discharge deficits with the deficits found by inactivating MD relay neurons.

Another way that spreading inactivation might have affected our results was to cause the bilateral deficits in saccadic reaction time and percent correct that we found as well as the occasional lethargy that we seemed to induce. We suspect that these systemic deficits were due to slightly decreased levels of arousal caused by muscimol seeping up the penetration shaft, around the cannula, to affect the overlying reticular nucleus of the thalamus that helps control sleep-wake transitions (McCormick 2002; Steriade 2003).

AMOUNT OF MUSCIMOL NEEDED. We injected 2.1 $\mu$ l of muscimol on average (Table 1), but it is important to note that this was the volume required to cause the effect that we used for verifying successful injection: an increase in current threshold for evoking saccades (see METHODS). The volume required to impair corollary discharge may well have been less. For example, the very similar deficits shown in Fig. 5, $A-D$, occurred during muscimol injections of 2.0, 1.6, 3.0, and $1.6 \mu \mathrm{l}$ respectively. This suggests that $\leq 1.6 \mu \mathrm{l}$ was needed to disrupt corollary discharge, an amount comparable to that used in other studies employing similar methods as ours [needle delivery of muscimol at $0.1-0.2 \mu \mathrm{l} / 30 \mathrm{~s}$ and at a concentration of $5 \mu \mathrm{g} / \mu \mathrm{l}$; e.g., Shi et al. (1998) used 1-1.4 $\mu \mathrm{l}$ in the FEF and Aizawa and Wurtz (1998) used 0.3-1.5 $\mu$ l in the SC].

OTHER POSSIBLE FUNCTIONS OF THE PATHWAY. By concluding from these inactivation results that the SC-MD-FEF pathway plays a role in corollary discharge of saccades, we do not mean to imply that it has no other function. Our recordings showed that a variety of signals in addition to presaccadic activity are conveyed from SC to MD to FEF (for details, see Sommer and Wurtz 2004b). Most notably, the pathway is also rich with phasic visual responses, suggesting that MD inactivation may also cause visual disruptions. A systematic test of this hypothesis was beyond the goals of this study.

\section{Other lesion studies investigating corollary discharge in primates}

To our knowledge, the present study is the first to document the oculomotor consequences of inactivating MD in monkeys and the first to describe the effects of specifically blocking any putative corollary discharge pathway in any primate. The most similar prior study was an examination of central thalamic lesions in humans (Gaymard et al. 1994) that revealed deficits in the ability to internally monitor movements, including sac- 
cadic eye movements, using tasks very similar to the doublestep paradigm used here. The lesions involved the internal medullary lamina and the ventral lateral and ventral posterolateral nuclei, while apparently sparing MD. Nevertheless it is quite possible that the lesions did disrupt the ascending pathway from SC to FEF. Damage to lateral MD abutting the internal medullary lamina cannot be ruled out, and that is precisely where MD relay neurons in monkeys are concentrated (Sommer and Wurtz 2004b). Also, in humans it is not known exactly where the thalamic neurons that relay signals from SC to FEF are located; they might lie outside of MD. The FEF seems to sit more posteriorly in humans (in Brodmann's area 6) than in other primates (area 8), implying that human SC-to-FEF relay neurons may lie more laterally, e.g., in the internal medullary lamina or the ventrolateral nucleus (reviewed by Tehovnik et al. 2000).

Many other lesion studies have shown that various parts of the brain are involved in corollary discharge (see INTRODUCTION). Surprisingly, though, corollary discharge deficits have not yet been demonstrated after permanent lesions of the FEF in either monkeys (Collin and Cowey 1980) or humans (Gaymard et al. 1999; Heide et al. 1995; Rivaud et al. 1994). This does not necessarily mean that the FEF is uninvolved in corollary discharge, however. In the monkey study (Collin and Cowey 1980), the task was to report with a lever press when one or both of a pair of lights moved; the logic was that if corollary discharge were impaired, monkeys would mistakenly report that saccade-induced displacements in the retinal images of the lights were actual movements of the lights. This did not occur after FEF (or SC) lesions, but many reasons could explain this: immediate corollary discharge deficits may have recovered in the days between surgery and testing; production of fewer saccades (Schiller et al. 1980) may have hidden a corollary discharge deficit (eye movements were not quantified); and the presentation of two lights simultaneously might have allowed the monkeys to perform the task in large part by noting when one light moved relative to the other, obviating the need to use corollary discharge so that deficits in corollary discharge went untested.

In the human FEF lesion studies, investigators have found that second saccades of the double-step task are impaired by damage to parietal cortex but not by damage to the FEF (Gaymard et al. 1999; Heide et al. 1995; Rivaud et al. 1994). Again, postlesion recovery is an issue; subjects might recover better from FEF than parietal damage. In particular, lesions of either area might disrupt corollary discharge of saccades while parietal lesions may additionally disrupt cortical signals related to static eye position. Reversible inactivation of lateral intraparietal cortex in monkeys performing a double-step task causes severe disruptions of eye position information $(\mathrm{Li}$ and Andersen 2001), and neuronal activity modulated by eye position is common in parietal cortex (reviewed by Andersen and Gnadt 1989) but not in the FEF (e.g., Bruce and Goldberg 1985; Goldberg and Bruce 1990). In sum, subjects with parietal lesions may lose both corollary discharge and eye position signals, leaving them with no hope of internally monitoring their saccades, whereas those with FEF lesions might lose corollary discharge and yet gradually adapt by making use of still-available eye position signals.

\section{Levels of corollary discharge in the primate saccadic system}

von Holst and Mittelstaedt (1950) suggested that keeping track of one's movements was based on monitoring outputs to muscles, and hence their term efference copy. The SC-MDFEF pathway, however, is at least a couple synapses above this final efferent level, so we have used Sperry's term of corollary discharge that indicates the interaction of "motor patterns" with a "sensorium" without specifying where the interaction occurs (Sperry 1950). Corollary discharge may arise from many levels of the nervous system, and indeed Evarts (1971) emphasized that a multilevel corollary discharge network might be critical in coordinating movements as well as in interpreting sensory inputs. Adapting Evarts' ideas, we hypothesize that corollary discharge arises at multiple levels in the saccadic system, essentially forming a chain composed of links associated with particular circuits. From link to link up the chain, the corollary

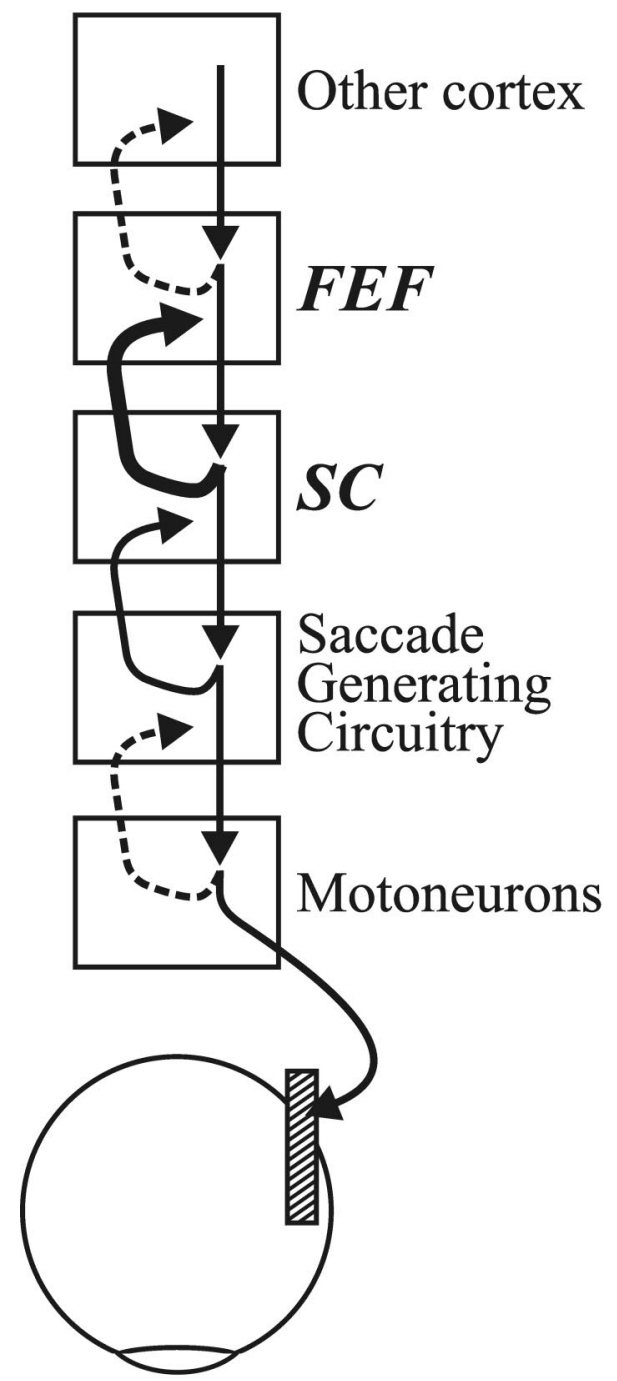

FIG. 14. Multiple levels of corollary discharge in the visuosaccadic system. Downward arrows depict gradual progression of the saccadic command, and upward arrows depict corollary discharge signals. The MD relay node is omitted from the SC-to-FEF feedback pathway for clarity. This is a highly simplified diagram that certainly will have to be revised as more ascending pathways are studied physiologically. In particular, anatomical evidence hints that many more corollary discharge pathways from subcortical regions up to cortex may be found. See DISCUSSION for details. At bottom are depicted the eye and an extraocular muscle. 
discharge represents the actual movement less and less. Figure 14 illustrates this concept.

WHAT THE MOTONEURONS TELL THE SACCADE GENERATING CIRCUITRY. Extraocular motoneurons may provide feedback (Fig. 14, lowest dashed arrow), in line with the original concept of von Holst and Mittelstaedt (1950), as some have been shown to emit intra-cranial collaterals (McCrea et al. 1986). Corollary discharge from motoneurons should represent the instantaneous state of the eye, i.e., its three-dimensional position and dynamics, and it probably influences the pontine and midbrain areas collectively referred to as the saccade generating circuitry. It is doubtful, however, that this information reaches cerebral cortex (reviewed by Bridgeman 1995). When motoneurons are stimulated to evoke a saccade just prior to a visually guided saccade, monkeys fail to compensate for the evoked saccade; they errantly make visually guided saccades as if the eyes had not moved (Mays and Sparks 1981; Schiller and Sandell 1983). The stimulation seems to fail at creating a corollary discharge signal that can inform the monkey about the evoked saccade. In contrast, monkeys do compensate for evoked saccades if the SC or parts of the pons are stimulated (Guthrie et al. 1983; Sparks et al. 1987).

WHAT THE SACCADE GENERATING CIRCUITRY TELLS THE SC. Just above the motoneuron level is the saccade generating circuitry, and the importance of corollary discharge in this network (Fig. 14, thin solid upward arrow) is well known (Robinson 1975). The number of corollary discharge paths in this network, where they originate and end, and the signals they carry are still a matter of debate and modeling. It is generally agreed that the signals represent saccadic dynamics, e.g., speed, and that the feedback reaches the SC (Keller and Edelman 1994; Keller et al. 1996a; Soetedjo et al. 2002).

WHAT THE SC TELLS THE FEF. While it is clear that many corollary discharge paths may ascend via thalamus to cortex (Steriade et al. 1997; Tanaka 2003), the one that is best understood at this point is that running from SC to FEF (Fig. 14, bold solid arrow). The anatomy of this pathway is well established (Lynch et al. 1994), some of its signals and functions were described in the present experiments, and a long history of research on the source of the pathway, the SC, provides valuable insight as to the nature of the corollary discharge signal sent to the FEF. This latter point is discussed in detail in the final DISCUSSION section.

WHAT THE FEF TELLS OTHER CORTICAL AREAS. The highest level of the chain in Fig. 14 depicts a hypothesis that corollary discharge may course from FEF to other areas of cortex. The FEF is well situated to be a gateway of corollary discharge, as it receives corollary discharge from the $\mathrm{SC}$, it may create its own corollary discharge of saccadic instructions that it sends downstream, and it sends efferents to wide areas of the prefrontal, dorsomedial frontal, parietal, and temporal lobes (Leichnetz and Goldberg 1988; Schall 1997). An apparent conundrum, however, is why the FEF-itself an apparent generator of saccadic commands-would even need to receive corollary discharge from the SC. This may be explained by the fact that presaccadic activity in the SC is more tightly linked to saccade generation than that in the FEF (reviewed by Sommer and Wurtz 2004a; Wurtz et al. 2001). The FEF gains more detailed and credible information as to when and where a saccade will go by listening to the SC than by relying only on its own internal signals.

That the FEF may distribute corollary discharge to the rest of cortex is consistent with the classic view of Teuber (1966) that frontal cortex in general plays such a role. We do not presume that the FEF is the only such gateway of corollary discharge to the rest of cortex, but it seems well suited to be one such gateway.

\section{Role of the SC-MD-FEF pathway in corollary discharge}

There are two key questions one must ask when analyzing any corollary discharge signal: what is the signal a corollary of and how does the recipient structure use it? First, because we know that the neurons projecting from SC to MD are similar to those in the overall population of neurons in the SC intermediate layers (Sommer and Wurtz 2004b), we can infer what is represented by the signals sent into the SC-MD-FEF pathway based on what is known about SC neurons in general. In this paper and the accompanying one (Sommer and Wurtz 2004b), we have used the shorthand terminology of saying that presaccadic activity in the SC-MD-FEF pathway encodes where a saccade will go. This is true when saccades are made to stationary visual targets, but if the actual saccade differs from that specified by the target, it becomes clear that the SC saccadic command is in target, not motor, coordinates (Frens and Van Opstal 1997; Goossens and Van Opstal 2000; Keller et al. 1996b; Stanford and Sparks 1994). The SC activity still represents a saccadic command, as it is intimately tied with executing the saccade (e.g., Everling et al. 1998; Sparks 1978) and is present with spontaneous saccades in darkness, sans visual targets (Wurtz and Goldberg 1971); it is just that, spatially, the command encodes the retinotopic location to achieve, not the exact saccadic trajectory that will be made. This is probably adequate for the cortex because the discrepancy between the SC representation of the saccade and the actual saccade would be negligible during the most typical natural behavior of inspecting a visual scene.

Another important aspect of the SC saccadic signal is its two-dimensional nature: it encodes direction and amplitude but not torsion (Hepp et al. 1993). The brain, however, seems to use corollary discharge of all three dimensions of eye movement (Medendorp et al. 2002). Corollary discharge of torsion therefore must arise from outside of the SC.

Finally, we must consider how the FEF uses the corollary discharge signals that it gets from the SC. Our recording and inactivation results indicate that these signals represent the vector of a planned saccade. Under the hypothesis that the FEF can perform vector subtraction (Goldberg and Bruce 1990), from this vector and one representing the visual target location relative to the fovea (carried by visually responsive FEF neurons), the second saccade in a double-step task could be calculated. Similar calculations could contribute to more complex behaviors as well (e.g., triple-step sequences) (Tian et al. 2000). The FEF may also use SC-derived corollary discharge for purely visual purposes. The visual receptive fields of many FEF neurons shift prior to a saccade to the locations they would be expected to occupy after the saccade (Sommer and Wurtz 2003; Umeno and Goldberg 1997), and such presaccadically remapped receptive fields may mediate the perception of space constancy across saccades (Ross et al. 2001). Recent psycho- 
physical studies suggest that SC-derived corollary discharge contributes unequally to coordination of saccadic sequences as opposed to maintenance of visual stability, being more important for the latter than the former (Bahcall and Kowler 1999; Tanaka 2003). Future inactivation studies of the SC-MD-FEF pathway are needed to directly test this hypothesis.

\section{A C K N O W LED G MENTS}

We thank our colleagues in the Laboratory of Sensorimotor Research for helpful comments and M. K. Smith for technical help with the inactivations.

\section{REFERENCES}

Aizawa H and Wurtz RH. Reversible inactivation of monkey superior colliculus. I. Curvature of saccadic trajectory. J Neurophysiol 79: 2082-2096, 1998.

Andersen R and Gnadt JW. Posterior parietal cortex. In: The Neurobiology of Saccadic Eye Movements, Reviews of Oculomotor Research, edited by Wurtz RH and Goldberg ME. Amsterdam: Elsevier, 1989, vol. III, p. 315-336.

Angel RW. Barognosis in a patient with hemiataxia. Ann Neurol 7: 73-77, 1980.

Bahcall DO and Kowler E. Illusory shifts in visual direction accompany adaptation of saccadic eye movements. Nature 400: 864-866, 1999.

Baizer JS, Kralj-Hans I, and Glickstein M. Cerebellar lesions and prism adaptation in macaque monkeys. J Neurophysiol 81: 1960-1965, 1999.

Becker W and Jürgens R. An analysis of the saccadic system by means of double step stimuli. Vision Res 19: 967-983, 1979.

Blakemore SJ, Wolpert D, and Frith C. Why can't you tickle yourself? Neuroreport 11: R11-16, 2000

Bridgeman B. A review of the role of efference copy in sensory and oculomotor control systems. Ann Biomed Eng 23: 409-422, 1995.

Bruce CJ and Goldberg ME. Primate frontal eye fields. I. Single neurons discharging before saccades. J Neurophysiol 53: 603-635, 1985.

Collin NG and Cowey A. The effect of ablation of frontal eye-fields and superior colliculi on visual stability and movement discrimination in Rhesus monkeys. Exp Brain Res 40: 251-260, 1980.

Crist CF, Yamasaki DSG, Komatsu H, and Wurtz RH. A grid system and a microsyringe for single cell recording. J Neurosci Methods 26: 117-122, 1988.

Dias EC and Segraves MA. Muscimol-induced inactivation of monkey frontal eye field: effects on visually and memory-guided saccades. $J$ Neurophysiol 81: 2191-2214, 1999.

Duhamel J-R, Colby CL, and Goldberg ME. The updating of the representation of visual space in parietal cortex by intended eye movements. Science 255: 90-92, 1992a.

Duhamel J-R, Goldberg ME, FitzGibbon EJ, Sirigu A, and Grafman J. Saccadic dysmetria in a patient with a right frontoparietal lesion: the importance of corollary discharge for accurate spatial behavior. Brain 115: 1387-1402, 1992b.

Edelman JA and Goldberg ME. Dependence of saccade-related activity in the primate superior colliculus on visual target presence. J Neurophysiol 86: 676-691, 2001.

Evarts EV. Central control of movement. V. Feedback and corollary discharge: a merging of the concepts. Neurosci Res Program Bull 9: 86-112, 1971 .

Everling S, Dorris MC, and Munoz DP. Reflex suppression in the antisaccade task is dependent on prestimulus neural processes. J Neurophysiol 80: 1584-1589, 1998.

Everling S and Munoz DP. Neuronal correlates for preparatory set associated with pro-saccades and anti-saccades in the primate frontal eye field. $\mathrm{J} \mathrm{Neu}$ rosci 20: 387-400, 2000.

Feinberg I and Guazzelli M. Schizophrenia-a disorder of the corollary discharge systems that integrate the motor systems of thought with the sensory systems of consciousness. Br J Psychiatry 174: 196-204, 1999.

Ford JM, Mathalon DH, Heinks T, Kalba S, Faustman WO, and Roth WT. Neurophysiological evidence of corollary discharge dysfunction in schizophrenia. Am J Psychiatry 158: 2069-2071, 2001.

Frens MA and Van Opstal AJ. Monkey superior colliculus activity during short-term saccadic adaptation. Brain Res Bull 43: 473-483, 1997.

Gaymard B, Ploner CJ, Rivaud-Pechoux S, and Pierrot-Deseilligny C. The frontal eye field is involved in spatial short-term memory but not in reflexive saccade inhibition. Exp Brain Res 129: 288-301, 1999.
Gaymard B, Rivaud S, and Pierrot-Deseilligny C. Impairment of extraretinal eye position signals after central thalamic lesions in humans. Exp Brain Res 102: 1-9, 1994.

Gnadt JW, Bracewell RM, and Andersen RA. Sensorimotor transformation during eye movements to remembered visual targets. Vision Res 31: 693$715,1991$.

Goldberg ME and Bruce CJ. Primate frontal eye fields. III. Maintenance of a spatially accurate saccade signal. J Neurophysiol 64: 489-508, 1990.

Goossens HHLM and Van Opstal AJ. Blink-perturbed saccades in monkey. II. Superior colliculus activity. J Neurophysiol 83: 3430-3452, 2000.

Guthrie BL, Porter JD, and Sparks DL. Corollary discharge provides accurate eye position information to the oculomotor system. Science 221: 1193-1195, 1983.

Haarmeier T, Thier P, Repnow M, and Petersen D. False perception of motion in a patient who cannot compensate for eye movements. Nature 389: 849-852, 1997.

Hallett PE and Lightstone AD. Saccadic eye movements toward stimuli triggered by prior saccades. Vision Res 16: 88-106, 1976.

Heide W, Blankenburg M, Zimmermann E, and Kompf D. Cortical control of double-step saccades: implications for spatial orientation. Ann Neurol 38: 739-748, 1995.

Hepp K, Van Opstal AJ, Straumann D, Hess BJ, and Henn V. Monkey superior colliculus represents rapid eye movements in a two-dimensional motor map. J Neurophysiol 69: 965-979, 1993.

Hikosaka $\mathrm{O}$ and Wurtz RH. Modification of saccadic eye movements by GABA-related substances. I. Effect of muscimol and bicuculline in monkey superior colliculus. J Neurophysiol 53: 266-291, 1985.

Keller EL and Edelman JA. Use of interrupted saccade paradigm to study spatial and temporal dynamics of saccadic burst cells in superior colliculus in monkey. J Neurophysiol 72: 2754-2770, 1994.

Keller EL, Gandhi NJ, and Shieh JM. Endpoint accuracy in saccades interrupted by stimulation in the omnipause region in monkey. Vis Neurosci 13: 1059-1067, 1996a.

Keller EL, Gandhi NJ, and Weir PT. Discharge of superior collicular neurons during saccades made to moving targets. J Neurophysiol 76: 3573 3577, $1996 b$.

Kowler E. The role of visual and cognitive processes in the control of eye movement. In: Eye Movements and their Role in Visual and Cognitive Processes, edited by Kowler E. Amsterdam: Elsevier, 1990, p. 1-63.

Leichnetz GR and Goldberg ME. Higher centers concerned with eye movement and visual attention: cerebral cortex and thalamus. In: Neuroanatomy of the Oculomotor System, edited by Büttner-Ennever JA. Amsterdam: Elsevier, 1988, p. 365-429.

Lewis RF, Zee DS, Hayman MR, and Tamargo RJ. Oculomotor function in the rhesus monkey after deafferentation of the extraocular muscles. Exp Brain Res 141: 349-358, 2001.

Li CS and Andersen RA. Inactivation of macaque lateral intraparietal area delays initiation of the second saccade predominantly from contralesional eye positions in a double-saccade task. Exp Brain Res 137: 45-57, 2001.

Lomber SG. The advantages and limitations of permanent or reversible deactivation techniques in the assessment of neural function. $J$ Neurosci Methods 86: 109-117, 1999.

Lynch JC, Hoover JE, and Strick PL. Input to the primate frontal eye field from the substantia nigra, superior colliculus, and dentate nucleus demonstrated by transneuronal transport. Exp Brain Res 100: 181-186, 1994.

Mays LE and Sparks DL. Dissociation of visual and saccade-related responses in superior colliculus neurons. J Neurophysiol 43: 207-232, 1980.

Mays LE and Sparks DL. The localization of saccade targets using a combination of retinal and eye position information. In: Progress in Oculomotor Research, edited by Fuchs A and Becker W. New York: Elsevier/ North Holland, 1981, p. 39-47.

McCormick DA. Cortical and subcortical generators of normal and abnormal rhythmicity. Int Rev Neurobiol 49: 99-114, 2002.

McCrea RA, Strassman A, and Highstein SM. Morphology and physiology of abducens motoneurons and internuclear neurons intracellularly injected with horseradish peroxidase in alert squirrel monkeys. J Comp Neurol 243: 291-308, 1986.

Medendorp WP, Smith MA, Tweed DB, and Crawford JD. Rotational remapping in human spatial memory during eye and head motion. $J \mathrm{Neu}$ rosci 22: 1-4, 2002.

Munoz DP, Guitton D and Pélisson D. Control of orienting gaze shifts by the tectoreticulospinal system in the head-free cat. III. Spatiotemporal characteristics of phasic motor discharges. J Neurophysiol 66: 1642-1666, 1991. 
Poulet JF and Hedwig B. A corollary discharge maintains auditory sensitivity during sound production. Nature 418: 872-876, 2002.

Rafal RD. Neglect. Curr Opin Neurobiol 4: 231-236, 1994.

Reppas JB, Usrey WM, and Reid RC. Saccadic eye movements modulate visual responses in the lateral geniculate nucleus. Neuron 35: 961-974, 2002.

Richmond BJ and Wurtz RH. Vision during saccadic eye movements. II. A corollary discharge to monkey superior colliculus. J Neurophysiol 43: 1156-1167, 1980.

Rivaud S, Muri RM, Gaymard B, Vermersch AI, and Pierrot-Deseilligny C. Eye movement disorders after frontal eye field lesions in humans. Exp Brain Res 102: 110-120, 1994.

Robinson DA. Oculomotor control signals. In: Basic Mechanisms of Ocular Motility and Their Clinical Implications, edited by Lennerstrand G and Bach-y-Rita P. Oxford, UK: Pergamon, 1975, p. 337-374.

Ross J, Concetta Morrone M, Goldberg ME, and Burr DC. Changes in visual perception at the time of saccades. Trends Neurosci 24: 113-121, 2001.

Schall JD. Visuomotor areas of the frontal lobe. In: Cerebral Cortex, edited by Rockland K, Kaas JH, and Peters A. New York: Plenum, 1997, p. 527-638.

Schiller PH and Sandell JH. Interactions between visually and electrically elicited saccades before and after superior colliculus and frontal eye field ablations in the rhesus monkey. Exp Brain Res 49: 381-392, 1983.

Schiller PH, True SD, and Conway JL. Deficits in eye movements following frontal eye field and superior colliculus ablations. J Neurophysiol 44: 11751189, 1980.

Schlag-Rey M and Schlag J. The central thalamus. In: The Neurobiology of Saccadic Eye Movements, Reviews of Oculomotor Research, edited by Wurtz RH and Goldberg ME. Amsterdam: Elsevier, 1989, vol. III, p. 378-381.

Segraves MA. Activity of monkey frontal eye field neurons projecting to oculomotor regions of the pons. J Neurophysiol 68: 1967-1985, 1992.

Segraves MA and Goldberg ME. Functional properties of corticotectal neurons in the monkey's frontal eye field. J Neurophysiol 58: 1387-1419, 1987.

Shi D, Friedman HR, and Bruce CJ. Deficits in smooth-pursuit eye movements after muscimol inactivation within the primate's frontal eye field. J Neurophysiol 80: 458-464, 1998.

Soetedjo R, Kaneko CRS, and Fuchs AF. Evidence that the superior colliculus participates in the feedback control of saccadic eye movements. J Neurophysiol 87: 679-695, 2002.

Sommer MA and Tehovnik EJ. Reversible inactivation of macaque frontal eye field. Exp Brain Res 116: 229-249, 1997.

Sommer MA and Tehovnik EJ. Reversible inactivation of macaque dorsomedial frontal cortex: effects on saccades and fixations. Exp Brain Res 124: 429-446, 1999.

Sommer MA and Wurtz RH. Composition and topographic organization of signals sent from the frontal eye field to the superior colliculus. J Neurophysiol 83: 1979-2001, 2000.

Sommer MA and Wurtz RH. Frontal eye field sends delay activity related to movement, memory, and vision to the superior colliculus. J Neurophysiol 85: 1673-1685, 2001.

Sommer MA and Wurtz RH. A pathway in primate brain for internal monitoring of movements. Science 296: 1480-1482, 2002.

Sommer MA and Wurtz RH. The frontal eye field sends predictively remapped visual signals to the superior colliculus. J Vision 3(9): 146a; 10.1167/3.9.146, 2003.

Sommer MA and Wurtz RH. The dialogue between cerebral cortex and superior colliculus: implications for saccadic target selection and corollary discharge. In: The Visual Neurosciences, edited by Chalupa LM and Werner JS. Cambridge, MA: MIT Press, 2004a, vol. 2, p. 1466-1484.

Sommer MA and Wurtz RH. What the brain stem tells the frontal cortex. I. Oculomotor signals sent from superior colliculus to frontal eye field via mediodorsal thalamus. J Neurophysiol 91: 1381-1402, 2004 b.

Sparks DL. Functional properties of neurons in the monkey superior colliculus: coupling of neuronal activity and saccade onset. Brain Res 156: 1-16, 1978.

Sparks DL, Mays LE, and Porter JD. Eye movements induced by pontine stimulation: interaction with visually triggered saccades. J Neurophysiol 53: 300-318, 1987.

Sperry RW. Neural basis of the spontaneous optokinetic response produced by visual inversion. J Comp Physiol Psychol 43: 482-489, 1950.

Stanford TR, Freedman EG, and Sparks DL. Site and parameters of microstimulation: evidence for independent effects on the properties of saccades evoked from the primate superior colliculus. J Neurophysiol 76: 3360-3381, 1996.

Stanford TR and Sparks DL. Systematic errors for saccades to remembered targets: evidence for a dissociation between saccade metrics and activity in the superior colliculus. Vision Res 34: 93-106, 1994.

Steinbach MJ. Proprioceptive knowledge of eye position. Vision Res 27: 1737-1744, 1987.

Steriade M. The corticothalamic system in sleep. Front Biosci 8: D878-899, 2003.

Steriade M, Jones EG, and McCormick DA. Thalamus: Organisation and Function. Amsterdam: Elsevier, 1997, vol. 1, p. 31-174.

Tanaka M. Contribution of signals downstream from adaptation to saccade programming. J Neurophysiol 90: 2080-2086, 2003.

Tehovnik EJ, Sommer MA, Chou I-H, Slocum WM, and Schiller PH. Eye fields in the frontal lobes of primates. Brain Res Rev 32: 413-448, 2000.

Teuber H-L. The frontal lobes and their function: further observations on rodents, carnivores, subhuman primates, and man. Int J Neurol 5: 282-300, 1966.

Thiele A, Henning P, Kubischik M, and Hoffmann KP. Neural mechanisms of saccadic suppression. Science 295: 2460-2462, 2002.

Tian J, Schlag J, and Schlag-Rey M. Testing quasi-visual neurons in the monkey's frontal eye field with the triple-step paradigm. Exp Brain Res 130: 433-440, 2000.

Tolias AS, Moore T, Smirnakis SM, Tehovnik EJ, Siapas AG, and Schiller PH. Eye movements modulate visual receptive fields of V4 neurons. Neuron 29: 757-767, 2001.

Umeno MM and Goldberg ME. Spatial processing in the monkey frontal eye field. I. Predictive visual responses. J Neurophysiol 78: 1373-1383, 1997.

Versino M, Beltrami G, Uggetti C, and Cosi V. Auditory saccade impairment after central thalamus lesions. J Neurol Neurosurg Psychiatry 68: 234-237, 2000.

von Holst E and Mittelstaedt H. Das Reafferenzprinzip. Wechselwirkungen zwischen Zentralnervensystem und Peripherie. Naturwissenschaften 37: 464-476, 1950.

Weese GD, Phillips JM, and Brown VJ. Attentional orienting is impaired by unilateral lesions of the thalamic reticular nucleus in the rat. J Neurosci 19: 10135-10139, 1999.

Wurtz RH and Goldberg ME. Superior colliculus cell responses related to eye movements in awake monkeys. Science 171: 82-84, 1971.

Wurtz RH, Sommer MA, Paré M, and Ferraina S. Signal transformations from cerebral cortex to superior colliculus for the generation of saccades. Vision Res 41: 3399-3412, 2001. 